\title{
Phase Transitions, Minimal Surfaces and A Conjecture of De Giorgi ${ }^{1}$
}

\author{
O. Savin
}

\section{Introduction}

A central problem in the area of PDEs is the study of global solutions, that is solutions defined in the whole space. This problem arises naturally for example when one studies the possible types of behaviors a solution might have at a given point. Focusing near such a point by dilating the picture more and more, we end up with a PDE in the whole space. In other cases we might have a differential equation with oscillating terms on a small scale depending on a parameter $\varepsilon$. In order to understand the behavior at unit scale as the parameter $\varepsilon \rightarrow 0$ we often need to dilate the picture and solve some PDE in the whole space. As a consequence, the study of global solutions is crucial when dealing with singularities, local behavior and homogenization limits of PDEs.

In this paper we present symmetry results for global solutions to certain semilinear (or fully nonlinear) elliptic equations of the type

$$
\triangle u=f(u)
$$

which arise in the theory of phase transitions.

Let us briefly introduce the typical physical model for a phase transition. Imagine in a domain $\Omega$ we have a two-phase fluid whose density at a point $x$ we denote by $\rho(x)$. Assume its energy density is given by a double-well potential $W(\rho(x))$ say with minima at $\rho_{1}, \rho_{2}$ i.e.

$$
W\left(\rho_{1}\right)=W\left(\rho_{2}\right)=0, \quad W(s)>0 \quad \text { if } s \neq \rho_{1}, \rho_{2} .
$$

The densities $\rho_{1}$ and $\rho_{2}$ correspond to the stable fluid phases. Then the energy of the fluid is given by the integral

$$
\int_{\Omega} W(\rho(x)) d x .
$$

\footnotetext{
${ }^{1}$ The author was supported by N.S.F. Grant DMS-07-01037 and a Sloan Fellowship.
} 
This is not a satisfactory physical model since any density function $\rho(x)$ that takes only the values $\rho_{1}$ and $\rho_{2}$ minimizes the density energy. In particular the stable phases $\rho_{1}$ and $\rho_{2}$ could coexist along any complicated interface! The reason for this is because we ignored the kinetic energy term that takes into consideration interactions at small scales (such as friction) which penalizes the formation of unnecessary interfaces. The energy associated with the fluid in this case would be of the form

$$
J_{\varepsilon}(\rho)=\int_{\Omega} \frac{\varepsilon^{2}}{2}|\nabla \rho|^{2}+W(\rho) d x
$$

with $\varepsilon$ a small parameter. This is the typical energy modeling the phase separation phenomena within the van der Walls-Cahn-Hilliard theory (see [12]).

The presence of the gradient square term in the energy prevents instantaneous jumps from a region of density $\rho_{1}$ to a region of density $\rho_{2}$, and in general this stable states are only asymptotically attained by $\rho$. The transition region between the two phases occurs in a thin region of width comparable to $\varepsilon$. If we try to understand this transition at length-scale $\varepsilon$ we need to dilate the picture by a factor of $1 / \varepsilon$. Then the rescaled density $u(x):=\rho(\varepsilon x)$ minimizes the energy

$$
\int \frac{1}{2}|\nabla u|^{2}+W(u) d x
$$

and hence solves the Euler-Lagrange equation

$$
\triangle u=W^{\prime}(u)
$$

in the large domain $\Omega / \varepsilon$. On the other hand, we shall see in Section 2 that if we let $\varepsilon \rightarrow 0$ in the original domain $\Omega$, the transition region converges to a minimal surface inside $\Omega$, i.e. a surface of least area. This fact was already known to the school of De Giorgi in Pisa in the late 1970's, and De Giorgi conjectured that global solutions to the Euler-Lagrange equation above should have similar properties to something apparently unrelated, global minimal surfaces.

The goal of this paper is to present several results in both settings, phase transitions and minimal surfaces, together with their common underlying ideas. Special interest is given to flatness theorems and their application to

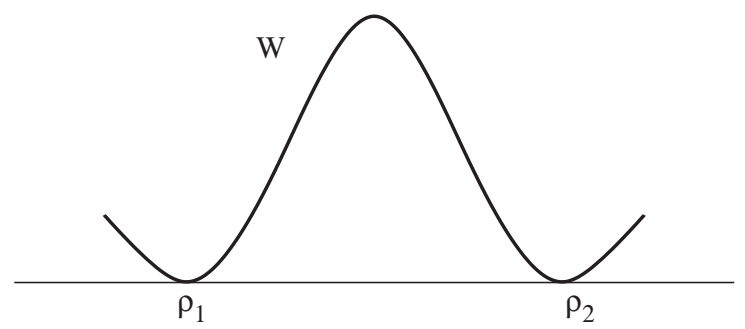

Figure 1. Double-well potential 
1D symmetry of global phase transitions and in particular to De Giorgi's conjecture. See Section 3 for the precise formulation of this conjecture.

We conclude our introduction with a brief description of the theory of minimal surfaces, with special emphasis on the ideas of De Giorgi.

Finding the surface of least area (minimal surface) among all surfaces bounded by a given curve is known as the Plateau problem. It is one of the oldest problems in mathematics and it has received a great deal of attention from the mathematical community. Plateau problem was solved independently by Douglas [27] and Rado [53,54] in the 1930's. Later, the theories of De Giorgi on perimeters [21-24], the one of Federer and Fleming $[\mathbf{3 3}, \mathbf{3 5}]$ on rectifiable currents and the one of Almgreen $[\mathbf{2}, \mathbf{3}]$ on varifolds were developed to deal with the higher dimensional case.

Let us explain the approach of De Giorgi. The key idea is to look at hypersurfaces in $\mathbb{R}^{n}$ as boundaries of sets. Precisely, for any measurable set $E$, we can define the perimeter of $E$ in a domain $\Omega \subset \mathbb{R}^{n}$ (or the area of $\partial E$ in $\Omega$ ) as the total variation of the characteristic function of $E, \chi_{E}$, in $\Omega$

$$
P_{\Omega}(E)=\int_{\Omega}\left|\nabla \chi_{E}\right|=\sup _{|g| \leq 1}\left|\int_{E} \operatorname{div} g d x\right|,
$$

where the supremum is taken over all vector fields $g \in C_{0}^{1}(\Omega)$ (with compact support in $\Omega$ ) with $|g| \leq 1$. From Green's theorem we see that this notion of area of $\partial E$ coincides with the usual one whenever $\partial E$ is a $C^{1}$ hypersurface. The advantage of defining the perimeter for a larger class of sets is the compactness in $L^{1}$ of sets with finite perimeter. Then it is not difficult to show existence to the Plateau problem in this context of minimal boundaries. It is much more difficult to prove that the sets so obtained are actually smooth hypersurfaces except possibly for a closed singular set of small Hausdorff dimension.

One understands the behavior of such a minimal surface in a neighborhood of a point $x_{0}$ by using the blow-up technique explained at the beginning. Dilating the picture more and more we end up with a limiting minimal surface defined in the whole space. What can be said about global minimal surfaces? Simons [59] proved that the only global minimal surfaces are the hyperplanes in dimension $n \leq 7$. Moreover, Bombieri, De Giorgi and Giusti $[\mathbf{1 1}]$ showed that the symmetric cone in $\mathbb{R}^{8}$

$$
\left\{x \in \mathbb{R}^{8}: x_{1}^{2}+x_{2}^{2}+x_{3}^{2}+x_{4}^{2}=x_{5}^{2}+x_{6}^{2}+x_{7}^{2}+x_{8}^{2}\right\}
$$

known as Simons cone is minimal. This is the first example of a minimal surface that has a singularity (at the origin). Going back to the original picture, Simons result says that at least in low dimensions the minimal surface is approximated well by a plane (it is flat) in a small neighborhood of $x_{0}$. On the other hand, a theorem of De Giorgi that holds in any dimension says that flat minimal surfaces in $B_{r}\left(x_{0}\right)$ are analytic in $B_{r / 2}\left(x_{0}\right)$. This "flatness theorem" gives smoothness near the points that have a planar blow-up limit. Thus, minimal surfaces are always smooth in dimension $n \leq 7$. 
In higher dimensions, it was shown by Federer [34] that they are smooth everywhere except on a small singular set of Hausdorff dimension $n-8$.

Minimal surfaces that are graphs in some direction are called minimal graphs. It turns out that minimal graphs are smooth in any dimension. The question wether the only global minimal graphs are planar is known as the Bernstein problem, due to Bernstein [10] (1915) which answered affirmatively the question in $\mathbb{R}^{3}$. Bombieri, De Giorgi and Giusti [11] showed that the graph assumption gains one extra dimension with respect to the minimal surface results mentioned earlier: minimal graphs are planar in dimension $n \leq 8$, and there are non-planar minimal graphs in $\mathbb{R}^{9}$.

The paper is organized as follows. In Section 2 we give the precise relation between minimal surfaces and level sets of minimizers of $J_{\varepsilon}$. In Section 3 we state the conjecture of De Giorgi together with other related problems. In Section 4 we prove density estimates in both settings. In Section 5 and 6 we give a new proof of the flatness theorem of De Giorgi for minimal surfaces which is based on Harnack inequality. In Sections 7 to 9 we extend these ideas to phase transitions and obtain the corresponding flatness theorem. In Section 10 and 11 we discuss $1 D$ symmetry for global phase transitions. Finally in Section 12 we present similar results that are available for fully nonlinear equations.

\section{Phase transitions and minimal surfaces}

We consider the Ginzburg-Landau energy functional

$$
J(u, \Omega)=\int_{\Omega} \frac{1}{2}|\nabla u|^{2}+W(u) d x, \quad|u| \leq 1
$$

with $W$ a double-well potential with minima at 1 and -1 satisfying

$$
\begin{gathered}
W \in C^{2}([-1,1]), \quad W(-1)=W(1)=0, \quad W>0 \text { on }(-1,1), \\
W^{\prime}(-1)=W^{\prime}(1)=0, \quad W^{\prime \prime}(-1)>0, \quad W^{\prime \prime}(1)>0 .
\end{gathered}
$$

The classical double-well potential $W$ to have in mind is

$$
W(s)=\frac{1}{4}\left(1-s^{2}\right)^{2} .
$$

We mention that all the results of this paper apply also to more general potentials $W$ that do not necessarily satisfy the conditions above. Such an example is the discontinuous potential $W(s)=\chi_{(-1,1)}(s)$, where $\chi_{(-1,1)}$ represents the characteristic function of the interval $(-1,1)$. In this case the physical motivation comes from a problem associated with jet fluid flows (see $[4])$.

In this section we explain the close relation between minimal surfaces and level sets of minimizers of $J$. 
Consider a minimizer $u$ of $J$ which is bounded by -1 and 1 . We say that $u$ is a minimizer in the (possible unbounded) set $\Omega$ if its energy increases locally under any compact perturbation, i.e. for any open set $A$ relatively compact in $\Omega$,

$$
J(u, A) \leq J(u+\varphi, A) \quad \forall \varphi \in H_{0}^{1}(A) .
$$

A minimizer in whole $\mathbb{R}^{n}$ is called a global minimizer.

The behavior of $u$ in large domains is given by the behavior of the rescaled functions $u_{\varepsilon}$ in $B_{1}$,

$$
u_{\varepsilon}(x):=u\left(\frac{x}{\varepsilon}\right) .
$$

If $u$ minimizes $J$ in the ball $B_{\frac{1}{\varepsilon}}$ then $u_{\varepsilon}$ minimizes the rescaled energy $J_{\varepsilon}$ in $B_{1}$

$$
J_{\varepsilon}\left(v, B_{1}\right):=\int_{B_{1}} \frac{\varepsilon}{2}|\nabla v|^{2}+\frac{1}{\varepsilon} W(v) d x .
$$

We continue with a heuristic discussion about minimizers of $J_{\varepsilon}$. For a given function $v$, the main contribution in $J_{\varepsilon}(v)$ comes from the potential energy which is minimized when $v$ equals either 1 or -1 . Instant jumps from a region where $v=1$ to a region where $v=-1$ are not allowed since the kinetic energy $\int \frac{\varepsilon}{2}|\nabla v|^{2}$ would become infinite. However, it is useful to notice that if instead of the Dirichlet integral we would have the BV norm $\int \varepsilon|\nabla v|$ then such jumps are allowed and in this case the energy is minimized when the jumps occur along a minimal surface.

Clearly

$$
\int_{B_{1}} \frac{\varepsilon}{2}|\nabla v|^{2}+\frac{1}{\varepsilon} W(v) d x \geq \int_{B_{1}} \sqrt{2 W(v)}|\nabla v| d x
$$

and using the co-area formula

$$
|\nabla v| d x=d_{\mathcal{H}^{n-1}(\{v=s\})} d s
$$

the inequality becomes

$$
J_{\varepsilon}(v) \geq \int_{-1}^{1} \sqrt{2 W(s)} \mathcal{H}^{n-1}(\{v=s\}) d s .
$$

The energy $J_{\varepsilon}$ is then minimized by the function $v$ if every level set is a minimal surface and we have equality in the inequality above i.e.

$$
|\nabla v|=\frac{1}{\varepsilon} \sqrt{2 W(v)} .
$$

This last equality gives

$$
v(x)=g_{0}\left(\frac{d_{\Gamma}(x)}{\varepsilon}\right),
$$




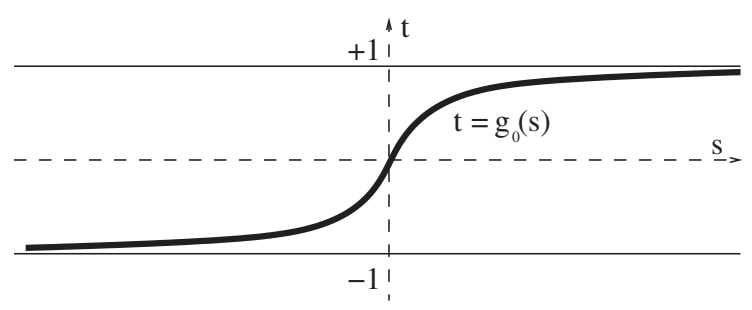

Figure 2. One-dimensional solution

where $d_{\Gamma}(x)$ represents the sign distance to the 0 level set $\Gamma:=\{v=0\}$ and $g_{0}$ is the solution to the ODE

$$
g_{0}^{\prime}=\sqrt{2 W\left(g_{0}\right)}, \quad g_{0}(0)=0 .
$$

The function $g_{0}$ is in fact the unique minimizer of $J$ in $1 \mathrm{D}$ which is increasing.

In general the level sets of a function $v$ as above cannot be all minimal surfaces. However, if for example the 0 level set $\Gamma$ is minimal then the $s$ level sets are essentially minimal as long as $s$ is not too close to \pm 1 and $\varepsilon$ is small. On the other hand when $s$ is close to \pm 1 the weight $\sqrt{2 W(s)} d s$ becomes negligible. Thus such a function is "almost" a minimizer for $J_{\varepsilon}$. This suggests that the level sets of minimizers of $J_{\varepsilon}$ converge to a minimal surface as $\varepsilon \rightarrow 0$.

The asymptotic behavior of $u_{\varepsilon}$ was first studied by Modica and Mortola [51] and by Modica [45] within the framework of $\Gamma$-convergence. Later Modica [47], Sternberg [60] and many authors $[6,36,43,49,52,61]$ generalized these results for minimizers with volume constraint.

Here we present the result of Modica in [45].

Theorem 2.1 (Modica). Let $u_{\varepsilon}$ be minimizers for the energies $J_{\varepsilon}\left(\cdot, B_{1}\right)$. There exists a sequence $u_{\varepsilon_{k}}$ such that

$$
u_{\varepsilon_{k}} \rightarrow \chi_{E}-\chi_{E^{c}} \quad \text { in } L_{l o c}^{1}\left(B_{1}\right)
$$

where $E$ is a set with minimal perimeter in $B_{1}$. Moreover, if $A$ is an open set, relatively compact in $B_{1}$, such that

$$
\int_{\partial A}\left|D \chi_{E}\right|=0
$$

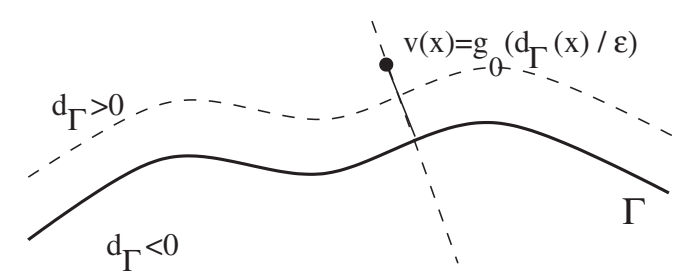

Figure 3. Approximate minimizer 
then

$$
\lim _{m \rightarrow \infty} J_{\varepsilon_{k}}\left(u_{\varepsilon_{k}}, A\right)=P_{A}(E) \int_{-1}^{1} \sqrt{2 W(s)} d s .
$$

Let us recall the notion of perimeter $P_{\Omega}(E)$ and minimal perimeter that appear in the theorem above. The perimeter of $E$ in $\Omega \subset \mathbb{R}^{n}$ is defined as

$$
P_{\Omega}(E)=\sup \left|\int_{E} \operatorname{div} g d x\right|,
$$

where the supremum is taken over all vector fields $g \in C_{0}^{1}(\Omega)$ with $\|g\| \leq 1$. When $\Omega$ is the whole $\mathbb{R}^{n}$ we use the shorter notation

$$
P(E)=P_{\mathbb{R}^{n}}(E) .
$$

We say that $E$ is a set with minimal perimeter in $\Omega$ or shortly $\partial E$ is a minimal surface in $\Omega$ if, for every open set $A \subset \Omega$, relatively compact in $\Omega$

$$
P_{A}(E) \leq P_{A}(F),
$$

whenever $E$ and $F$ coincide outside a compact set included in $A$.

From the heuristic discussion before the theorem of Modica we see that the converse is also true at least in the case when the minimal surface $\partial E$ is unique i.e. given a minimal surface $\partial E$ we can find a sequence of minimizers $u_{\varepsilon}$ converging to $\chi_{E}-\chi_{\mathcal{C} E}$. Therefore minimal surfaces and minimizers of $J_{\varepsilon}$ should have similar properties at least for small $\varepsilon$.

In Section 4 we obtain density estimates for level sets of minimizers. As a consequence, the convergence of $u_{\varepsilon_{k}}$ in Theorem 2.1 is in fact stronger than $L_{l o c}^{1}$ i.e. the level sets of $u_{\varepsilon_{k}}$ converge uniformly on compact sets to $\partial E$.

If the functions $u_{\varepsilon_{k}}$ are all rescalings of a global minimizer $u$ of $J$, then the blow-down sets

$$
\left\{u_{\varepsilon_{k}}=0\right\}=\varepsilon_{k}\{u=0\}
$$

converge uniformly to a minimal surface. It is a difficult problem to understand how well the original level set $\{u=0\}$ can be approximated by a minimal surface. For example in the case when $\partial E=\left\{x_{n}=0\right\}$ it is not clear wether or not $\{u=0\}$ stays at a bounded distance from $\partial E$. Conversely,

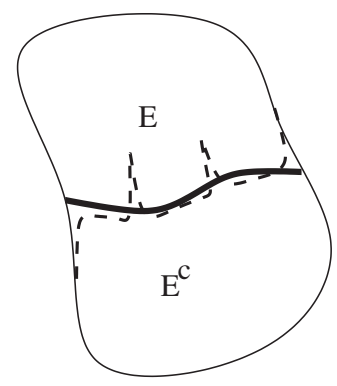

Figure 4. Convergence of $u_{\varepsilon}$. 
given a nontrivial global minimal surface $\partial E$ it is not evident if there exists at least one minimizer for which $\{u=0\}$ stays at a bounded distance from $\partial E$. Such an example was given by Del Pino, Kowalczyk and Wei $[\mathbf{2 6}]$ in dimension 9 which is the first dimension for which a nontrivial global minimal graph exists.

\section{De Giorgi's conjecture}

In view of the connection between minimal surfaces and minimizers of $J$, De Giorgi [20] made in 1978 the following conjecture about bounded solutions of a global semilinear equation:

Conjecture (De Giorgi). Let $u \in C^{2}\left(\mathbb{R}^{n}\right)$ be a solution of

$$
\triangle u=u^{3}-u
$$

such that

$$
|u|<1, \quad u_{x_{n}}>0
$$

in whole $\mathbb{R}^{n}$. Is it true that all the level sets of $u$ are hyperplanes, at least if $n \leq 8$ ?

The conjecture is sometimes referred as "the $\varepsilon$ version of the Bernstein problem for minimal graphs". This relation with the Bernstein problem is the reason why $n \leq 8$ appears in the conjecture.

De Giorgi's conjecture is often considered with the additional natural hypothesis which is coherent with the phase transition setting

$$
\lim _{x_{n} \rightarrow \pm \infty} u\left(x^{\prime}, x_{n}\right)= \pm 1
$$

The monotonicity $u_{x_{n}}>0$ implies that the level sets of $u$ are graphs in the $e_{n}$ direction whereas the condition (3.1) says that these graphs are defined over whole $\mathbb{R}^{n-1}$. Under the much stronger assumption that the limits in (3.1) are uniform in $x^{\prime}$, the conjecture is known as the Gibbons conjecture. In this case the level sets of $u$ are bounded graphs in the $x_{n}$ direction.

Gibbons conjecture was first proved for $n \leq 3$ by Ghoussoub and Gui in [37] and then for all dimensions $n$ independently by Barlow, Bass and Gui [7], Berestycki, Hamel and Monneau [9] and Farina [29].

The first positive partial result on the De Giorgi conjecture was established in 1980 by Modica and Mortola [50]. They proved the conjecture in dimension $n=2$ under the additional hypothesis that the level sets $\{u=s\}$ are equi-Lipschitz in the $x_{2}$ direction. Their proof used a Liouville-type theorem for elliptic equations in divergence form, due to Serrin, for the bounded ratio

$$
\sigma:=\frac{u_{x_{1}}}{u_{x_{2}}}
$$


In 1997 Ghoussoub and Gui [37] proved De Giorgi conjecture for $n=2$. They used a different Liouville-type theorem for $\sigma$ developed by Berestycki, Caffarelli and Nirenberg [8] for the study of symmetry properties of positive solutions of semilinear elliptic equations in half spaces. This theorem does not require for $\sigma$ to be bounded, but rather a compatibility condition between the growth of $\sigma$ and the degeneracy of the coefficients of the equation. Using similar techniques, Ambrosio and Cabre [5] extended these results to dimension $n=3$. Also, Ghoussoub and Gui showed in [38] that the conjecture is true for $n=4$ or $n=5$ for a special class of solutions that satisfy an anti-symmetry condition. We shall discuss the methods described above in Section 11.

In 2003 the conjecture was proved by the author in [55] for $n \leq 8$ under the additional hypothesis (3.1). One of the goals of this expository paper is to explain the main ideas of [55] (see Sections 7-10). The methods developed do not depend so much on the particular dimension but rather they allow one to obtain rigidity results for global phase transitions as a consequence of the results for minimal surfaces.

Recently, Del Pino Kowalczyk and Wei [26] gave a counterexample to the De Giorgi's conjecture in dimension 9. This counterexample satisfies also the limiting assumption (3.1). The construction is based on careful perturbations of the level sets around the nonplanar minimal graph in $\mathbb{R}^{9}$ constructed by De Giorgi, Bombieri and Giusti.

De Giorgi's conjecture refers to one analogy between phase transitions and minimal surfaces, i.e. the Bernstein problem. There are many other similar questions that can be posed. For example, is it true that minimizers of $J$ are one-dimensional at least for $n \leq 7$ ? If the level sets are Lipschitz graphs, is it true that the solution must be one-dimensional in any dimension? What if the level sets satisfy only a one-sided bound? What happens if we consider a fully nonlinear equation instead of the semilinear one? We answer these questions in Section 10-12.

Referring to the questions above, when the level sets are assumed to be Lipschitz graphs, it was first shown by Barlow, Bass and Gui [7] that $u$ is one-dimensional with probabilistic methods. Different proofs were given by the author in [55] and by Caffarelli and Cordoba in [18]. Also, we mention that Cabre and Terra in [13] are investigating the existence of nontrivial symmetric global minimizers in dimension 8, which are analogous to Simons cone.

There are further similarities between the theory of phase transitions and the theory of minimal surfaces besides the rigidity of global solutions. For example level sets of minimizers of $J$ satisfy uniform density estimates (see next section). Also, there is a monotonicity formula for minimizers which is due to Modica (see [48]) and reads

$$
\Phi(r)=\frac{J_{B_{r}}(u)}{r^{n-1}}
$$


is increasing in $r$. However, this monotonicity formula is not as powerful as the one in the minimal surface case since $\Phi$ is not scale invariant and it is not constant for the one-dimensional solutions.

Caffarelli and Cordoba developed in [17] a theory for phase transitions similar to the one of De Giorgi for Lipschitz minimal graphs. Also, as we will see in Section 7, phase transitions satisfy an improvement of flatness theorem which is key in our analysis and corresponds to De Giorgi flatness theorem for minimal surfaces.

\section{Density estimates}

In this section we prove density estimates for minimal surfaces and phase transitions. These are crucial in both theories since they allow us to pass from convergence in measure ( $L^{1}$ convergence) to uniform convergence.

4.1. Minimal surfaces. Let $E$ be a measurable set in $\mathbb{R}^{n}$.

We say that $0 \in \partial E$ (in measure sense) if for any $\varepsilon>0$ we have

$$
\left|B_{\varepsilon} \cap E\right|>0, \quad\left|B_{\varepsilon} \cap \mathcal{C} E\right|>0,
$$

where $\mathcal{C} E$ denotes the complement of $E$ and $|E|$ the Lebesgue measure of $E$. It is not difficult to see that $\partial E$ in measure sense is a closed set.

TheOREM 4.1 (Density estimates). Assume that $E$ has minimal perimeter in $B_{1}$ and $0 \in \partial E$. There exists a constant $c>0$ depending only on the dimension $n$ such that for all $r \in(0,1)$

$$
\left|E \cap B_{r}\right|>c r^{n}, \quad\left|\mathcal{C} E \cap B_{r}\right|>c r^{n} .
$$

Proof. Minimality implies

$$
P_{B_{1}}(E) \leq P_{B_{1}}\left(E \backslash B_{r}\right)
$$

or

$$
P_{B_{r}}(E) \leq \mathcal{H}^{n-1}\left(E \cap \partial B_{r}\right)
$$

hence

$$
P\left(E \cap B_{r}\right) \leq 2 \mathcal{H}^{n-1}\left(E \cap \partial B_{r}\right) .
$$

We denote $V(r)=\left|B_{r} \cap E\right|$ and we use the isoperimetric inequality

$$
c V(r)^{\frac{n-1}{n}} \leq P\left(E \cap B_{r}\right)
$$

to obtain

$$
c V(r)^{\frac{n-1}{n}} \leq V^{\prime}(r)
$$

or

$$
c \leq\left(V^{\frac{1}{n}}(r)\right)^{\prime}
$$




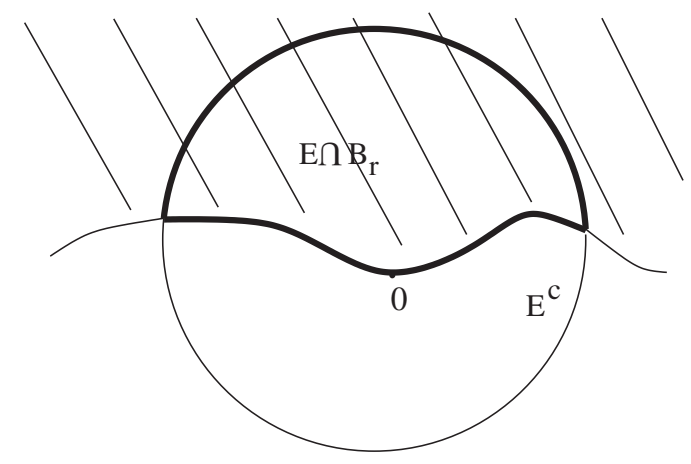

Figure 5. Density estimate

Here we used that $V(r)>0$ for all $r>0$ since $0 \in \partial E$. We integrate the last inequality and obtain the first density estimate. The second estimate follows by considering $\mathcal{C} E$.

Next we recall the standard compactness theorem for sets with minimal perimeter. A proof can be found in the book of Giusti [40].

THEOREM 4.2 (Compactness). If $E_{n}$ is a sequence of minimal sets in $\Omega$ then there exists a subsequence $E_{n_{k}}$ that converges to a minimal set $E$ i.e.

$$
\chi_{E_{n_{k}}} \rightarrow \chi_{E} \quad \text { in } L_{l o c}^{1}(\Omega) .
$$

The density estimates imply that the minimal surfaces $\partial E_{k}$ converge in Hausdorff sense to $\partial E$ on any compact set of $\Omega$. Indeed, if for some large $k$ there exists a point $x_{0} \in \partial E_{k}$ at distance $\delta$ from $\partial E$, then from the density estimates we find

$$
\int_{B_{\delta}\left(x_{0}\right)}\left|\chi_{E_{k}}-\chi_{E}\right| \geq c\left|B_{\delta}\right|,
$$

and we contradict the convergence of $E_{k}$ to $E$ in $L_{l o c}^{1}$.

As we explained in the introduction, an important example occurs when $\Omega=\mathbb{R}^{n}$ and the limiting minimal surface $\partial E$ is planar, say $\partial E=\left\{x_{n}=0\right\}$. Then, the uniform convergence implies that for any $\varepsilon>0$

$$
\partial E_{k} \cap B_{1} \subset\left\{\left|x_{n}\right|<\varepsilon\right\}
$$

for all large enough $k$.

4.2. Phase transitions. Next we present density estimates for level sets of minimizers

$$
J(u, \Omega)=\int_{\Omega} \frac{1}{2}|\nabla u|^{2}+W(u) d x, \quad|u| \leq 1 .
$$

which were obtained by Cordoba and Caffarelli [17]. In this setting the domain $\Omega$ is large and the density estimates hold for large enough balls. 


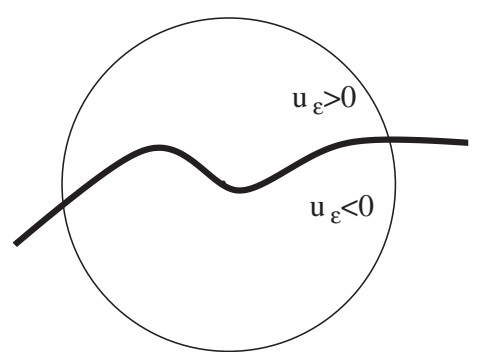

Figure 6. Density estimate for $u_{\varepsilon}$

Before we state the precise statement we first give a bound for the growth of $J\left(u, B_{r}\right)$.

Proposition 4.3. Assume $r \geq 1$ and $B_{r+2} \subset \Omega$. Then

$$
J\left(u, B_{r}\right) \leq C r^{n-1},
$$

where $C$ depends on $n$ and $W$.

ProOF. We compare $u$ with the compact perturbation

$$
v=\min \{u, h\} \quad \text { with } h(x):=\min \left\{(|x|-r)^{+}-1,1\right\},
$$

and obtain

$$
J\left(u, B_{r}\right) \leq J(u,\{u<v\}) \leq J(h,\{u<v\}) \leq J\left(h, B_{r+2}\right) \leq C r^{n-1} .
$$

TheOrem 4.4 (Caffarelli-Cordoba). Given $\alpha>-1, \beta<1$, if $u$ is a minimizer of $J$ in $B_{R}$ and $u(0) \geq \alpha$, then

$$
\left|\{u>\beta\} \cap B_{r}\right| \geq c r^{n}
$$

for $r \geq r_{0}(\alpha, \beta)$, where $c$ is a constant depending on $n$ and $W$.

Proof. The proof consists of two steps. In the first step we prove a weaker version when $\alpha$ and $\beta$ are close to -1 and in the second step we extend it to the general case. The ideas are similar to the minimal surface case but the proof is more involved. Let $\lambda_{0}$ be close to -1 so that $W$ is increasing in the interval $\left[-1, \lambda_{0}\right]$.

Step 1. Let $-1<\lambda \leq \lambda_{0}$. We prove the theorem with $\alpha=1+2 \lambda, \beta=\lambda$ and with the constant $c$ depending also on $\lambda$.

For each $r \geq 1$ denote by

$$
V(r):=\left|\{u>\lambda\} \cap B_{r}\right|, \quad a(r):=\int_{B_{r}} W(u) d x .
$$


We want to deduce a discrete differential inequality involving $V$ and $a$ that resembles the one for minimal surfaces. We use as comparison function in $B_{r}$ the radially symmetric function

$$
v:=-1+2 e^{|x|-r} .
$$

The test function $v$ was chosen such that $v=1$ on $\partial B_{r}$ and satisfies

$$
|\nabla v|^{2} \leq C\left(W(v)+\chi_{\{v>0\}}\right) \leq C\left(W(v)+\chi_{\{v>\lambda\}}\right),
$$

for some constant $C$ depending on $W$.

Denote by $K$ the closure of the open set $\{u>v\}$, and clearly

$$
J(u, K) \leq J(v, K) .
$$

As in Section 2, the coarea formula gives

$$
\int_{-1}^{1} \mathcal{H}^{n-1}(\{w=s\} \cap K) \sqrt{2 W(s)} d s \leq J(w, K),
$$

for any function $w$ with values in $[-1,1]$.

For each $s \in(-1,1)$ denote (see Figure 7 )

$$
E_{s}:=\{v<s<u\} .
$$

We have

$$
\partial E_{s}=(\{u=s\} \cup\{v=s\}) \cap K
$$

and by the isoperimetric inequality

$$
\left|E_{s}\right|^{\frac{n-1}{n}} \leq \mathcal{H}^{n-1}(\{u=s\} \cap K)+\mathcal{H}^{n-1}(\{v=s\} \cap K) .
$$

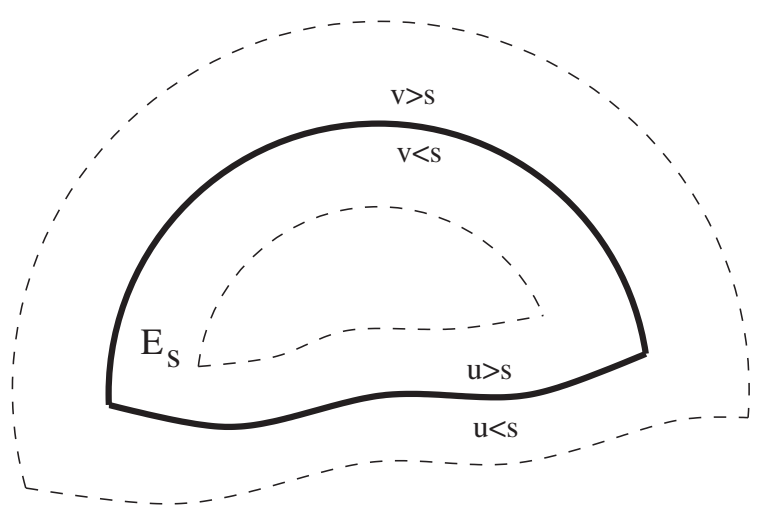

Figure 7. The set $E_{s}$ 
Multiplying with $\sqrt{2 W(s)}$ and integrating in $s$ we obtain

$$
\int_{-1}^{1}\left|E_{s}\right|^{\frac{n-1}{n}} \sqrt{2 W(s)} d s \leq J(u, K)+J(v, K) \leq 2 J(v, K) .
$$

When $s<\lambda$, we have the inclusion $\{u>\lambda\} \cap B_{r} \subset E_{s}$ thus

$$
V(r)=\left|\{u>\lambda\} \cap B_{r}\right| \leq\left|E_{s}\right| .
$$

The above integral inequality implies

$$
c(\lambda) V(r)^{\frac{n-1}{n}} \leq J(v, K),
$$

with $c(\lambda)>0$ a small constant that depends also on $\lambda$.

Also $J(u, K) \leq J(v, K)$ gives

$$
a(r) \leq J(v, K)
$$

hence

$$
c(\lambda)\left(a(r)+V(r)^{\frac{n-1}{n}}\right) \leq J(v, K) .
$$

Next we estimate the right hand side $J(v, K)$ by splitting $K$ into two domains

$$
K_{1}:=B_{r-T} \cap K, \quad K_{2}:=K \cap\left(B_{r} \backslash B_{r-T}\right),
$$

where $T$ is a large constant to be made precise later. Clearly,

$$
J\left(v, K_{1}\right) \leq J\left(v, B_{r-T}\right) \leq C e^{-T} r^{n-1} .
$$

For the second integral we use the inequality on $|\nabla v|^{2}$ together with $v \leq u$ on $K$ and $W$ increasing on $[0, \lambda]$ :

$$
\begin{aligned}
J\left(v, K_{2}\right) & \leq C \int_{K_{2}} W(v)+\chi_{\{v>\lambda\}} d x \\
& \leq C \int_{K_{2}} W(u)+\chi_{\{u>\lambda\}} d x \\
& \leq C(V(r)-V(r-T)+a(r)-a(r-T)) .
\end{aligned}
$$

In conclusion

$$
c(\lambda)\left(a(r)+V(r)^{\frac{n-1}{n}}\right) \leq V(r)+a(r)-(V(r-T)+a(r-T))+e^{-T} r^{n-1} .
$$

From the Euler-Lagrange equation for the minimizer we find that $u$ is Lipschitz in $B_{1}$ with the Lipschitz norm depending only on $W$. Since

$$
u(0) \geq \alpha=1+2 \lambda
$$

we find that $V(1) \geq \mu_{0}(\lambda)$. This and the inequality above easily implies that the sequence

$$
d_{k}:=a(k T)+V(k T)
$$


satisfies a discrete differential inequality

$$
c(\lambda) d_{k}^{\frac{n-1}{n}} \leq d_{k+1}-d_{k}+e^{-T} T^{n-1} k^{n-1}, \quad d_{1} \geq \mu_{0}(\lambda) .
$$

The coefficient of $k^{n-1}$ tends to 0 as $T \rightarrow \infty$. It is straightforward to check that

$$
d_{k} \geq \mu_{1} k^{n}
$$

for some small $\mu_{1}(\lambda)$ provided that $T$ is chosen large depending on $\lambda$. This gives

$$
V(r)+a(r) \geq c(\lambda) r^{n}
$$

The energy bound (4.1) implies $a(r) \leq C r^{n-1}$, thus

$$
V(r) \geq c(\lambda) r^{n} \quad \text { if } r \geq 1,
$$

and Step 1 is proved.

Step 2. We remove the assumptions on $\alpha, \beta$ and $c$ by increasing the initial radius $r \geq r_{1}(\alpha, \beta)$.

Since $u(0) \geq \alpha$ we can find a small $\lambda$ depending on $\alpha$ with $-1<\lambda \leq \lambda_{0}$, and $\lambda \leq(\alpha-1) / 2$. By Step 1 there exists $c(\alpha)>0$ such that for all $r \geq 1$

$$
\left|\{u>\lambda\} \cap B_{r}\right| \geq c(\alpha) r^{n} .
$$

On the other hand, the energy bound (4.1) gives,

$$
\left|\{\beta \geq u>\lambda\} \cap B_{r}\right| \leq C(\lambda, \beta) J\left(u, B_{r}\right) \leq C(\lambda, \beta) r^{n-1}
$$

hence

$$
\left|\{u>\beta\} \cap B_{r}\right| \geq c(\alpha) r^{n}
$$

for all $r>r_{0}(\alpha, \beta)$.

Finally we remark that in (4.2) we can take the constant to be independent of $\alpha$ by possibly increasing $r_{0}(\alpha, \beta)$.

Indeed, by (4.2) we know that there exists a constant $C(\alpha)>0$ and a point $x_{0} \in B_{C(\alpha)}$ such that $u\left(x_{0}\right) \geq 0$. Now we can apply (4.2) with $\alpha=0$ by taking $x_{0}$ to be the origin, and obtain

$$
\left|\{u>\beta\} \cap B_{r}\left(x_{0}\right)\right| \geq c r^{n}
$$

for all $r \geq r_{0}(\beta)$, where $c$ depends only on $W$. In conclusion

$$
\left|\{u>\beta\} \cap B_{r}\right| \geq c r^{n}
$$

for all $r>r_{1}(\alpha, \beta)$ and the theorem is proved. 
4.3. Asymptotic behavior. As a consequence of the density estimates we obtain that the level sets of $u$ are asymptotically flat at $\infty$ at least in low dimensions. If $u: \mathbb{R}^{n} \rightarrow[-1,1]$ is a global minimizer then, by Modica's theorem, the rescalings

$$
u_{\varepsilon_{k}}(x)=u\left(x / \varepsilon_{k}\right)
$$

satisfy

$$
u_{\varepsilon_{k}} \rightarrow \chi_{E}-\chi_{\mathcal{C} E} \quad \text { in } L_{l o c}^{1}\left(\mathbb{R}^{n}\right),
$$

with $\partial E$ a global minimal surface. Then the density estimates imply, as in the minimal surface case, that the level sets

$$
\left\{u_{\varepsilon_{k}}=0\right\}=\varepsilon_{k}\{u=0\}
$$

converge uniformly on compact sets to $\partial E$. Since $\partial E$ is a global minimal surface, then $\partial E$ is a hyperplane if $n \leq 7$, say

$$
\partial E=\left\{x_{n}=0\right\} .
$$

Then $\{u=0\}$ is asymptotically flat at $\infty$, i.e. there exist sequences $\theta_{k}, l_{k}$ with $l_{k} \rightarrow \infty, \theta_{k} / l_{k} \rightarrow 0$ such that

$$
\{u=0\} \cap B_{l_{k}} \subset\left\{\left|x_{n}\right| \leq \theta_{k}\right\} .
$$

\section{Flatness theorem of De Giorgi}

In next two sections we give a new proof of the flatness theorem of De Giorgi that we mentioned in the introduction. The methods seem to be quite general and they can be applied to other equations or variational problems. Later we will use the same ideas to prove the corresponding flatness theorem for phase transitions.

The precise statement of the flatness theorem is the following.

Theorem 5.1 (De Giorgi). Assume $E$ is minimal in $B_{1}, 0 \in \partial E$ and

$$
\partial E \cap B_{1} \subset\left\{\left|x_{n}\right| \leq \varepsilon_{0}\right\}
$$

with $\varepsilon_{0}(n)$ small depending only on $n$. Then $\partial E$ is an analytic surface in $B_{1 / 2}$.

The difficulty of this theorem lies in the fact that $\partial E$ cannot be written as a graph. De Giorgi's proof relies on the monotonicity formula for minimal surfaces and the approximation of $\partial E$ by harmonic functions (see [40]). We will give a proof based on Harnack inequality and viscosity solutions methods. First we give a different version of the theorem above known as improvement of flatness.

TheOrem 5.2 (Improvement of flatness). Assume $E$ is minimal in $B_{1}$, $0 \in \partial E$ and

$$
\partial E \cap B_{1} \subset\left\{\left|x_{n}\right| \leq \varepsilon\right\}
$$




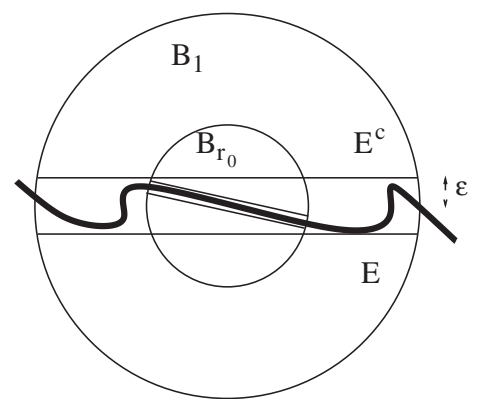

FiguRE 8. Improvement of flatness

with $\varepsilon \leq \varepsilon_{0}(n)$. Then there exists a unit vector $\nu_{1}$ such that

$$
\partial E \cap B_{r_{0}} \subset\left\{\left|x \cdot \nu_{1}\right| \leq \frac{\varepsilon}{2} r_{0}\right\},
$$

where $r_{0}$ is a small universal constant.

This theorem implies that $\partial E$ is a $C^{1, \alpha}$ graph in $B_{3 / 4}$. To see this we apply the theorem inductively and we obtain unit vectors $\nu_{k}$ such that

$$
\partial E \cap B_{r_{0}^{k}} \subset\left\{\left|x \cdot \nu_{k}\right| \leq \frac{\varepsilon}{2^{k}} r_{0}^{k}\right\} \text {. }
$$

This gives

$$
\left|\nu_{k+1}-\nu_{k}\right| \leq C\left(r_{0}\right) \frac{\varepsilon}{2^{k}}
$$

hence $\nu_{k} \rightarrow \nu(0)$ and moreover

$$
\left|\nu_{k}-\nu(0)\right| \leq C \frac{\varepsilon}{2^{k}}
$$

We obtain

$$
\partial E \cap B_{r_{0}^{k}} \subset\left\{x \cdot \nu(0) \leq C \frac{\varepsilon}{2^{k}} r_{0}^{k}=C \varepsilon r_{0}^{k(1+\alpha)}\right\},
$$

which implies that $\partial E$ is a differentiable surface at 0 with normal $\nu(0)$. Applying this argument at all points in $\partial E \cap B_{3 / 4}$ we see that $\partial E$ is in fact a $C^{1, \alpha}$ surface.

Once the surface is a $C^{1, \alpha}$ graph, we apply Schauder's estimates (see [39]) for the minimal surface equation satisfied by the graph and obtain that in $B_{1 / 2}, \partial E$ is smooth and in fact analytic.

We will derive the improvement of flatness theorem from the following weaker result.

TheOrem 5.3 (Harnack inequality). Assume $E$ is minimal in $B_{1}$ and

$$
\partial E \cap B_{1} \subset\left\{\left|x_{n}\right| \leq \varepsilon\right\}
$$

with $\varepsilon \leq \varepsilon_{1}(n)$. Then

$$
\partial E \cap B_{1 / 2} \subset\left\{\left|x_{n}\right| \leq \varepsilon(1-\eta)\right\},
$$

where $\eta>0$ is a small universal constant. 
Before we proceed we need to introduce the notion of viscosity solutions for boundaries of sets.

Definition. We say that the boundary of a set $\partial F$ satisfies the mean curvature equation

$$
M\left(D^{2} v, \nabla v\right):=\left(1+|\nabla v|^{2}\right) \triangle v-(\nabla v)^{T} D^{2} v \nabla v=0
$$

in the viscosity sense if for any smooth function $\varphi$ which has the subgraph

$$
S:=\left\{x_{n}<\varphi\left(x^{\prime}\right)\right\}
$$

included in $F$ or $\mathcal{C} F$ in a small ball $B_{\rho}(Y)$ around some point

$$
Y=\left(y^{\prime}, y_{n}\right) \in \partial F \cap \partial S
$$

we have

$$
M\left(D^{2} \varphi, \nabla \varphi\right) \leq 0
$$

If we consider the supergraph of $\varphi$ then the opposite inequality holds.

LEMMA 5.4. If $\partial E$ is a minimal surface then $\partial E$ satisfies the mean curvature equation in the viscosity sense.

A proof of this lemma can be found in [16]. The idea is that if $E$ contains the subgraph of $\varphi$ and $M\left(D^{2} \varphi, \nabla \varphi\right)>0$, then we can decrease the perimeter by adding to $E$ the small domain

$$
\left\{\varphi\left(x^{\prime}\right)<x_{n}<\varphi\left(x^{\prime}\right)+\varepsilon^{2}-\varepsilon\left|x^{\prime}\right|^{2}\right\} .
$$

Next we explain why Harnack inequality gives the improvement of flatness theorem. The main reason is that Harnack inequality allows us to approximate a flat minimal surface by a harmonic function.

Theorem 5.3 implies Theorem 5.2

The proof is by compactness. Assume by contradiction the statement of Theorem 5.2 is not true. Then we can find a sequence of minimal surfaces $\partial E_{k}$ which satisfy the hypothesis

$$
0 \in \partial E_{k} \cap B_{1} \subset\left\{\left|x_{n}\right|<\varepsilon_{k}\right\}
$$

with $\varepsilon_{k} \rightarrow 0$ for which the conclusion does not hold.

At each point $x_{0} \in \partial E_{k} \cap B_{1 / 2}$ we apply Harnack inequality in $B_{1 / 2}\left(x_{0}\right)$ and obtain that

$$
\partial E_{k} \cap B_{1 / 4}\left(x_{0}\right) \subset\left\{\left|\left(x-x_{0}\right) \cdot e_{n}\right| \leq 2 \varepsilon_{k}(1-\eta)\right\} .
$$

We apply Harnack inequality repeatedly as long as the hypothesis is satisfied. We obtain that for all $m \geq 2$ such that

$$
\varepsilon_{k} 2^{m}(1-\eta)^{m-2}<\varepsilon_{1}(n)
$$

we have the inclusion

$$
\partial E_{k} \cap B_{2^{-m}}\left(x_{0}\right) \subset\left\{\left|\left(x-x_{0}\right) \cdot e_{n}\right| \leq 2 \varepsilon_{k}(1-\eta)^{m-1}\right\} .
$$


Clearly we can take $m \rightarrow \infty$ as $\varepsilon_{k} \rightarrow 0$.

We strech this picture by a factor $\varepsilon_{k}^{-1}$ in the $x_{n}$ direction. The sets

$$
A_{k}:=\left\{\left(x^{\prime}, \frac{x_{n}}{\varepsilon_{k}}\right) \mid\left(x^{\prime}, x_{n}\right) \in \partial E_{k} \cap B_{1}\right\}
$$

are included in $\left\{\left|x_{n}\right| \leq 1\right\}$. Moreover, for each $m$ as above

$$
A_{k} \cap\left\{\left|x^{\prime}-x_{0}^{\prime}\right| \leq 2^{1-m}\right\} \subset\left\{\left|\left(x-x_{0}\right) \cdot e_{n}\right| \leq 2(1-\eta)^{m-1}\right\} .
$$

This gives a Hölder modulus of continuity of $A_{k}$ outside a $C \varepsilon_{k}^{-1}$ neighborhood of 0 . By Arzela-Ascoli theorem we can assume that as $\varepsilon_{k} \rightarrow 0$, by passing if necessary to a subsequence, $A_{k}$ converges in Hausdorff distance to the graph of a Hölder continuous function $\left(x^{\prime}, w\left(x^{\prime}\right)\right)$ in $\left\{\left|x^{\prime}\right| \leq 1 / 2\right\}$.

Next we show that $w$ is harmonic in the viscosity sense, that is $w$ cannot be touched by below (above) at an interior point by a strict subharmonic (superharmonic) quadratic polynomial.

Assume $P\left(x^{\prime}\right)$ is a quadratic polynomial whose graph touches by below the graph of $w$ at some point. Then $A_{k}$ touches a translation of $P$ which implies that $\partial E_{k}$ touches the graph of $\varepsilon_{k} P\left(x^{\prime}\right)+c$ at some interior point. Clearly $\partial E_{k}$ satisfies the minimal surface equation in the viscosity sense hence

$$
M\left(\varepsilon_{k} D^{2} P, \varepsilon_{k} \nabla P\right)=\varepsilon_{k} \triangle P+\varepsilon_{k}^{3}\left(|\nabla P|^{2} \Delta P-(\nabla P)^{T} D^{2} P \nabla P\right) \leq 0
$$

at the contact point. We let $\varepsilon_{k} \rightarrow 0$ and obtain

$$
\triangle P \leq 0 .
$$

Since $w$ is harmonic, $w(0)=0$ (since $\left.0 \in A_{k}\right)$ and $|w| \leq 1$, we find that

$$
\left|w\left(x^{\prime}\right)-x^{\prime} \cdot \nabla w(0)\right| \leq \frac{r_{0}}{4} \quad \text { if }\left|x^{\prime}\right| \leq 2 r_{0},
$$

provided that $r_{0}$ is chosen small, universal. This easily implies

$$
A_{k} \cap\left\{\left|x^{\prime}\right| \leq \frac{3}{2} r_{0}\right\} \subset\left\{\left|x_{n}-x^{\prime} \cdot \nabla w(0)\right| \leq \frac{r_{0}}{3}\right\}
$$

for large $k$ hence,

$$
\partial E_{k} \cap B_{r_{0}} \subset\left\{\left|x \cdot \nu_{k}\right| \leq \frac{\varepsilon_{k}}{2} r_{0}\right\} \quad \text { for some }\left|\nu_{k}\right|=1 .
$$

Thus $\partial E_{k}$ satisfies the conclusion of the Theorem and we reach a contradiction. 


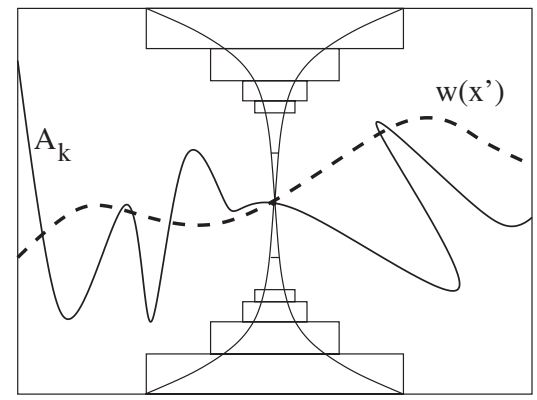

Figure 9. Convergence of $A_{k}$

\section{Harnack inequality}

In this section we will give a proof of Harnack inequality, Theorem 5.3. The ideas come from the proof of the classical Harnack inequality for uniformly elliptic second order linear equations due to Krylov and Safonov [44]. Let us briefly explain the key steps in their proof. First, they obtain an estimate in $L^{\infty}$ from one point to a set of points of fixed positive measure. Then, they iterate this step using Calderon-Zygmund decomposition and obtain $L^{\infty}$ estimates on a set of almost full measure, from which Harnack inequality can be deduced.

Of course these results do not apply directly in our case. The mean curvature equation $M\left(D^{2} v, \nabla v\right)=0$ is not uniformly elliptic since the ellipticity constants depend on $|\nabla v|$. Moreover, the set $\partial E$ cannot be written as a graph of a function $v$. However, the measure estimates still hold. This is because the set on which $L^{\infty}$ estimates hold is obtained as a contact set with a family of paraboloids that lie below the graph of $v$. Due to the flatness assumption, at the contact points the gradient of $v$ is small, and the equation becomes uniformly elliptic. Also, the minimality and flatness of $\partial E$ imply that from the measure point of view, $\partial E$ is almost a graph. Below we give the details. A systematic treatment of estimates for flat solutions to general elliptic equations can be found in [57].

First we prove a measure estimates for a general elliptic equation in $\mathbb{R}^{n}$.

$$
F\left(D^{2} v, D v\right)=0, \quad v: B_{1} \rightarrow \mathbb{R},
$$

with

$$
F: \mathcal{S} \times \mathbb{R}^{n} \rightarrow \mathbb{R}
$$

where $\mathcal{S}$ denotes the space of $n \times n$ symmetric matrices. We assume that $F$ is uniformly elliptic only in a neighborhood of $(0,0) \in \mathcal{S} \times \mathbb{R}^{n}$. Precisely, assume there exist $\delta>0$ small and $\lambda, \Lambda$ such that if $|p| \leq \delta$ and $N \geq 0$ is 
positive symmetric then

$$
\begin{aligned}
& F(0, p)=0, \\
& \lambda|N| \leq F(M+N, p)-F(M, p) \leq \Lambda|N| \quad \text { if }|M|,|N| \leq \delta, \\
& 0 \leq F(M+N, p)-F(M, p) \text { if }|M|<\delta .
\end{aligned}
$$

Clearly, these hypotheses are satisfied by the mean curvature equation.

We prove our estimates for supersolutions $v$ of the equation above. That is we assume $v$ is continuous, and any quadratic polynomial $P$ that touches $v$ by below at an interior point $x_{0}$ satisfies

$$
F\left(D^{2} P, D P\left(x_{0}\right)\right) \leq 0 .
$$

Proposition 6.1 (Estimate in measure). Let $v: B_{1} \rightarrow \mathbb{R}, v \geq 0$ be a viscosity supersolution to equation (6.1). Given $\mu>0$ small, there exist $\varepsilon$ small and $M$ large depending on $\mu$ and $n, \delta, \lambda, \Lambda$ such that if

$$
v(0) \leq \varepsilon,
$$

then

$$
\left|\{v>M v(0)\} \cap B_{1 / 3}\right|<\mu .
$$

In this section positive constants depending on $n, \lambda, \Lambda$ and $\delta$ are called universal constants.

For a small number $a>0$, we consider the paraboloids with center at $y \in \bar{B}_{1}$ and opening $-a$, i.e.

$$
-\frac{a}{2}|x-y|^{2}+\text { const }
$$

We slide them vertically from $-\infty$ till they touch the graph of $v$. We denote the set of interior contact points with $D_{a}$ (see Figure 10). Precisely,

$$
\begin{aligned}
D_{a}:= & \left\{z \in B_{1} \mid \exists y \in \bar{B}_{1} \quad \text { s.t. } v(z)+\frac{a}{2}|z-y|^{2} \leq v(x)\right. \\
& \left.+\frac{a}{2}|x-y|^{2} \quad \forall x \in B_{1}\right\} .
\end{aligned}
$$

We prove Proposition 6.1 by estimating the size of $D_{a}$. Notice that

$$
v(z) \leq v(0)+2 a, \quad \forall z \in D_{a} .
$$

First we obtain an estimate of the contact set of $z$ 's in terms of the set of centers $y$ that we start. This is a variant of Alexandrov-Bakelman-Pucci estimate for uniformly elliptic equations.

LEMmA 6.2 (ABP estimate). Let $a>0$ be small, $a \leq \delta / 2$, and let $F \subset \bar{B}_{1}$ be a closed set. For each $y \in F$, we slide the paraboloid of opening $-a$ and center $y$ by below till we touch the graph of $v$ for the first time. 


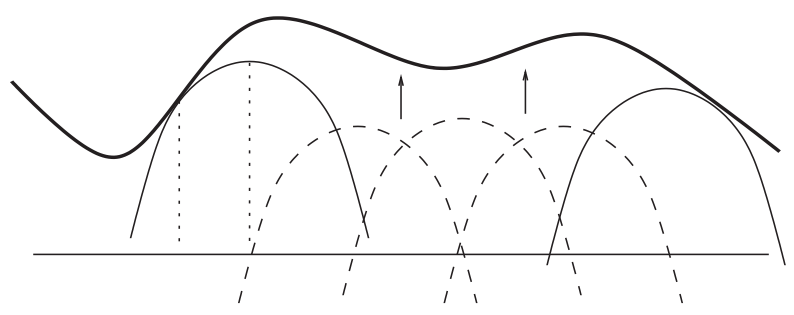

FiguRE 10. Sliding paraboloids

Suppose the set of all contact points, denoted by E, is included in a compact set of $B_{1}$. Then

$$
|E| \geq c|F|
$$

with $c>0$ a small universal constant.

Proof. Let us assume for simplicity that $v$ is smooth. Otherwise one needs to regularize $v$ using a standard inf-convolution.

At a contact point $z$, the corresponding center $y(z)$ is given by

$$
y(z)=z+\frac{1}{a} D v(z) .
$$

Since $y, z \in \bar{B}_{1}$ we see that $|D v(z)| \leq 2 a \leq \delta$. The differential map is

$$
D_{z} y=I+\frac{1}{a} D^{2} v(z)
$$

and moreover, since $v$ has at $z$ a tangent paraboloid of opening $-a$ by below,

$$
D^{2} v(z) \geq-a I
$$

Now we claim that the equation implies

$$
D^{2} v(z) \leq C a I
$$

with $C$ a large constant depending on $n, \delta, \lambda, \Lambda$. Indeed, otherwise

$$
D^{2} v(z) \geq C a e \otimes e-a I,
$$

for some unit vector $e$ hence

$$
F\left(D^{2} v(z), D v(z)\right) \geq F(C a e \otimes e-a I, p) \geq \lambda(C-1) a-(n-1) \Lambda a>0,
$$

and we contradict that $v$ is a supersolution.

In conclusion

$$
0 \leq D_{z} y \leq C I
$$

which gives

$$
|F|=\int_{E}\left|\operatorname{det} D_{z} y\right| d z \leq C|E|
$$


LEMma 6.3. There exist positive universal constants $C, c$ such that if $a \leq C^{-1} \delta$ and

$$
D_{a} \cap B_{r}\left(x_{0}\right) \neq \emptyset
$$

for some ball $\bar{B}_{r}\left(x_{0}\right) \subset B_{1}$, then

$$
\left|D_{C a} \cap B_{r / 8}\left(x_{0}\right)\right| \geq c r^{n} .
$$

Proof. Let

$$
z_{1} \in B_{r}\left(x_{0}\right) \cap D_{a},
$$

be a contact point and denote by $y_{1} \in \bar{B}_{1}$ the center of the tangent paraboloid

$$
P\left(x, y_{1}\right)=-\frac{a}{2}\left|x-y_{1}\right|^{2}+v\left(z_{1}\right)+\frac{a}{2}\left|z_{1}-y_{1}\right|^{2},
$$

that touches $v$ by below at $z_{1}$. First we find a point $x_{1} \in \bar{B}_{r / 16}\left(x_{0}\right)$ such that

$$
v\left(x_{1}\right)-P\left(x_{1}, y_{1}\right) \leq \operatorname{Car}^{2}, \quad C \text { universal. }
$$

Let $\phi: \bar{B}_{1} \rightarrow \mathbb{R}^{+}$be the radially symmetric continuous function

$$
\phi(x)= \begin{cases}\alpha^{-1}\left(|x|^{-\alpha}-1\right), & 1 / 16 \leq|x| \leq 1 \\ \alpha^{-1}\left(16^{\alpha}-1\right), & |x|<1 / 16 .\end{cases}
$$

where $\alpha$ is a large universal constant. Construct a function $\psi$ (see Figure 11) by adding a rescaling of the above function to the tangent paraboloid $P\left(x, y_{1}\right)$ i.e.

$$
\psi(x):=P\left(x, y_{1}\right)+a r^{2} \phi\left(\frac{x-x_{0}}{r}\right) .
$$

We claim that $\psi$ is a strict subsolution of our equation in the annular region

$$
\frac{r}{16}<\left|x-x_{0}\right|<r
$$

Indeed,

$$
|D \psi| \leq|D P|+|D \phi| \leq C a \leq \delta,
$$

and, by choosing $\alpha$ sufficiently large,

$$
\begin{aligned}
F\left(D^{2} \psi, D \psi\right) & =F\left(-a I+a D^{2} \phi, p\right) \\
& \geq \lambda\left((\alpha+1) t^{-\alpha-2}-1\right)-\Lambda(n-1)\left(1+t^{-\alpha-2}\right)>0
\end{aligned}
$$

where

$$
t=: \frac{\left|x-x_{0}\right|}{r}, \quad \frac{1}{16} \leq t \leq 1 .
$$




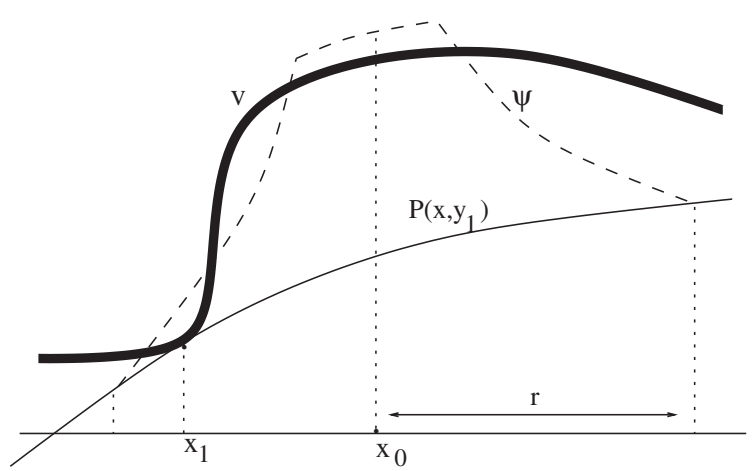

Figure 11. The function $\psi$

Now we slide the graph of $\psi$ from below till we touch the graph of $v$ for the first time. In other words we look for the point $x_{1}$ where the minimum

$$
\min _{\bar{B}_{r}\left(x_{0}\right)}(v-\psi)
$$

is realized. Notice that the minimum value is negative since

$$
v\left(z_{1}\right)-\psi\left(z_{1}\right)=P\left(z_{1}, y_{1}\right)-\psi\left(z_{1}\right)=-a r^{2} \phi\left(\frac{z_{1}-x_{0}}{r}\right)<0 .
$$

This implies that $x_{1}$ does not belong to $\partial B_{r}\left(x_{0}\right)$ because

$$
v(x)-\psi(x) \geq P\left(x, y_{1}\right)-P\left(x, y_{1}\right)=0 \quad \text { if } x \in \partial B_{r}\left(x_{0}\right) .
$$

Since $\phi$ is a strict subsolution in the annular region above we conclude that $x_{1} \in B_{r / 16}\left(x_{0}\right)$ and

$$
v\left(x_{1}\right)<\psi\left(x_{1}\right) \leq P\left(x_{1}, y_{1}\right)+\operatorname{Car}^{2}
$$

which proves (6.2).

Now we slide from below the family of paraboloids

$$
P\left(x, y_{1}\right)-C^{\prime} \frac{a}{2}|x-y|^{2}+c_{y}, \quad \text { with }\left|y-x_{1}\right| \leq r / 64
$$

till they become tangent to the graph of $v$. The opening of the above paraboloid is $-\left(C^{\prime}+1\right) a$ and the center

$$
\frac{C^{\prime}}{C^{\prime}+1} y+\frac{1}{C^{\prime}+1} y_{1} \text {. }
$$

The centers range over a ball of radius $\mathrm{cr}$ as $y \in B_{r / 64}\left(x_{1}\right)$. From (6.2) we find

$$
c_{y} \leq \operatorname{Car}^{2}+C^{\prime} \frac{a}{2}(r / 64)^{2}
$$


If $\left|x-x_{1}\right| \geq r / 16$ and $C^{\prime}$ is sufficiently large,

$$
\begin{aligned}
P\left(x, y_{1}\right)-C^{\prime} \frac{a}{2}|x-y|^{2}+c_{y} \leq & P\left(x, y_{1}\right)-C^{\prime} \frac{a}{2}(r / 32)^{2}+\operatorname{Car}^{2}+C^{\prime} \frac{a}{2}(r / 64)^{2} \\
& <P\left(x, y_{1}\right) \leq v(x) .
\end{aligned}
$$

Thus, the contact points are inside $B_{r / 16}\left(x_{1}\right) \subset B_{r / 8}\left(x_{0}\right)$. From the previous lemma we obtain

$$
\left|D_{\left(C^{\prime}+1\right) a} \cap B_{r / 8}\left(x_{0}\right)\right| \geq c r^{n},
$$

with $c$ small universal, and the lemma is proved.

Next we prove a simple measure covering lemma.

Lemma 6.4 (Covering lemma). Assume the closed sets $F_{k}$ satisfy

$$
F_{0} \subset F_{1} \subset F_{2} \cdots \subset \bar{B}_{1 / 3}, \quad F_{0} \neq \emptyset
$$

and for any $x, r$ such that

$$
\begin{gathered}
B_{r / 8}(x) \subset B_{1 / 3}, \quad \bar{B}_{r}(x) \subset B_{1}, \\
F_{k} \cap B_{r}(x) \neq \emptyset,
\end{gathered}
$$

then

$$
\left|F_{k+1} \cap B_{r / 8}(x)\right| \geq c r^{n} .
$$

Then

$$
\left|B_{1 / 3} \backslash F_{k}\right| \leq\left(1-c_{1}\right)^{k}\left|B_{1 / 3}\right|
$$

for some small constant $c_{1}$ depending on $c$.

Proof. Let $x_{0} \in B_{1 / 3} \backslash F_{k}$ and let

$$
r:=\operatorname{dist}\left(x_{0}, F_{k}\right) \text {. }
$$

We first prove that

$$
\left|F_{k+1} \cap B_{r / 3}\left(x_{0}\right)\right| \geq c_{1}\left|B_{r}\left(x_{0}\right) \cap B_{1 / 3}\right| .
$$

Let (see Figure 12)

$$
x_{1}:=x_{0}-\frac{r}{6} \frac{x_{0}}{\left|x_{0}\right|},
$$

and it is easy to check that

$$
B_{r / 6}\left(x_{1}\right) \subset B_{r / 3}\left(x_{0}\right) \cap B_{1 / 3}, \quad \bar{B}_{7 r / 6}\left(x_{1}\right) \subset B_{1} .
$$

Since

$$
\operatorname{dist}\left(x_{1}, F_{k}\right) \leq r+\frac{r}{6},
$$

we apply the hypothesis and conclude

$$
\left|F_{k+1} \cap B_{r / 6}\left(x_{1}\right)\right| \geq c r^{n} \geq c_{1}\left|B_{r}\left(x_{0}\right) \cap B_{1 / 3}\right|,
$$

which proves (6.3). 


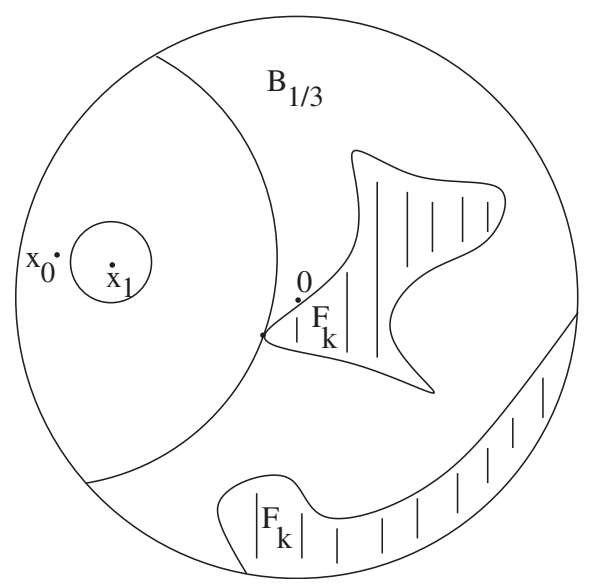

Figure 12. Covering of $\mathcal{C} F_{k}$

For each $x \in B_{1 / 3} \backslash F_{k}$ we let $r=\operatorname{dist}\left(x, F_{k}\right)$. From the family $B_{r}(x)$ we choose a Vitali subcover, i.e. balls $B_{r_{i}}\left(x_{i}\right)$ that cover $B_{1 / 3} \backslash D_{k}$ for which $B_{r_{i} / 3}\left(x_{i}\right)$ are disjoint.

We have

$$
\begin{aligned}
\left|B_{1 / 3} \backslash F_{k}\right| & \leq \sum\left|B_{r_{i}}\left(x_{i}\right) \cap B_{1 / 3}\right| \\
& \leq \sum c_{1}^{-1}\left|B_{r_{i} / 3}\left(x_{i}\right) \cap F_{k+1}\right| \leq c_{1}^{-1}\left|F_{k+1} \backslash F_{k}\right|,
\end{aligned}
$$

which implies

$$
\left|B_{1 / 3} \backslash F_{k+1}\right| \leq\left|B_{1 / 3} \backslash F_{k}\right|-\left|F_{k+1} \backslash F_{k}\right| \leq\left(1-c_{1}\right)\left|B_{1 / 3} \backslash F_{k}\right| .
$$

Proof of Proposition 6.1. Let

$$
a=20 v(0),
$$

and define

$$
F_{k}:=D_{C^{k} a} \cap \bar{B}_{1 / 3},
$$

where $C$ is the constant from Lemma 6.3. Since $v \geq 0$ in $B_{1}$, the paraboloid of opening $-a$ and center 0 touches the graph of $v$ for the first time in $\bar{B}_{1 / 3}$, hence $F_{0} \neq \emptyset$. Moreover, by Lemma 6.3 the hypothesis of the Covering lemma are satisfied as long as $C^{k} a \leq \delta$. Thus

$$
\left|B_{1 / 3} \backslash D_{C^{k} a}\right| \leq\left(1-c_{1}\right)^{k}\left|B_{1 / 3}\right|, \quad \text { if } C^{k} a \leq \delta .
$$

In the set $D_{C^{k} a}$ the function $v$ is less than $3 C^{k} a$, and we prove the proposition by first choosing $k$ large depending on $\mu$ and then $v(0) \leq \varepsilon$ small, so that $20 C^{k} \varepsilon \leq \delta$. 
Finally we can give the proof of Theorem 5.3 by applying the results above to the mean curvature equation in $\mathbb{R}^{n-1}$.

Proof of Theorem 5.3. Let $\partial E$ be a minimal surface in $B_{1}$ with

$$
0 \in \partial E \subset\left\{\left|x_{n}\right| \leq \varepsilon\right\} .
$$

Assume without loss of generality that in $B_{1}$ the set $E$ contains $\left\{x_{n}<-\varepsilon\right\}$ and is contained in $\left\{x_{n} \leq \varepsilon\right\}$. We view $\partial E$ as a multivalued graph over the $x^{\prime} \in \mathbb{R}^{n-1}$ that satisfies the mean curvature equation in the viscosity sense. We denote by $B_{r}^{\prime}\left(x^{\prime}\right)$ the $n-1$ dimensional ball of center $x^{\prime}$ and radius $r$ and by $\pi_{n}$ the projection along $e_{n}$ in $\mathbb{R}^{n-1}$.

It suffices to prove that

$$
\partial E \cap B_{1 / 10} \subset\left\{x_{n} \geq-\varepsilon(1-\eta)\right\}
$$

for some small constant $\eta>0$ depending on $n$. Assume by contradiction that there exists

$$
z \in \partial E \cap B_{1 / 10}, \quad \text { with } z_{n} \leq-\varepsilon(1-\eta) .
$$

Then we can use the argument in Proposition 6.1 to show the existence of a subset $D$ of contact points (see Figure 9)

$$
D \subset \partial E \cap\left\{\left|x^{\prime}-z^{\prime}\right|<3 / 10, \quad x_{n} \leq-\varepsilon+M \eta \varepsilon\right\}
$$

with

$$
\mathcal{H}^{n-1}\left(\pi_{n}(D)\right) \geq(1-\mu) \mathcal{H}^{n-1}\left(B_{3 / 10}^{\prime}\left(z^{\prime}\right)\right),
$$

with $\mu$ a small universal constant to be made precise later. We choose $\eta$ sufficiently small depending on $\mu$ such that $M \eta<1 / 2$. Then $D$ lies below the plane $x_{n}=-\varepsilon / 2$.

Next we use Lemma 6.2 to find another closed subset of contact points in $\partial E$ (see Figure 13),

$$
A \subset \partial E \cap B_{1 / 10} \cap\left\{x_{n} \geq-\varepsilon / 2\right\}
$$

so that

$$
\mathcal{H}^{n-1}\left(\pi_{n}(A)\right)>c_{0}
$$

with $c_{0}>0$ a small constant depending only on $n$. For this we slide paraboloids

$$
x_{n}=\frac{a}{2}\left|x^{\prime}-y^{\prime}\right|^{2}+\text { const }
$$

of opening $a=10^{3} \varepsilon$ and centers $\left|y^{\prime}\right| \leq 10^{-2}$ by above till they touch $\partial E$. Since $0 \in \partial E$, it is easy to check that the contact set $A$ is included in the set

$$
\left\{\left|x^{\prime}\right|<1 / 10, \quad-\varepsilon / 2 \leq x_{n} \leq \varepsilon\right\} .
$$

The bound on $\mathcal{H}^{n-1}\left(\pi_{n}(A)\right)$ follows from the measure estimate of Lemma 6.2 because the family of centers $y^{\prime}$ projects along $e_{n}$ into a set of fixed positive measure. 


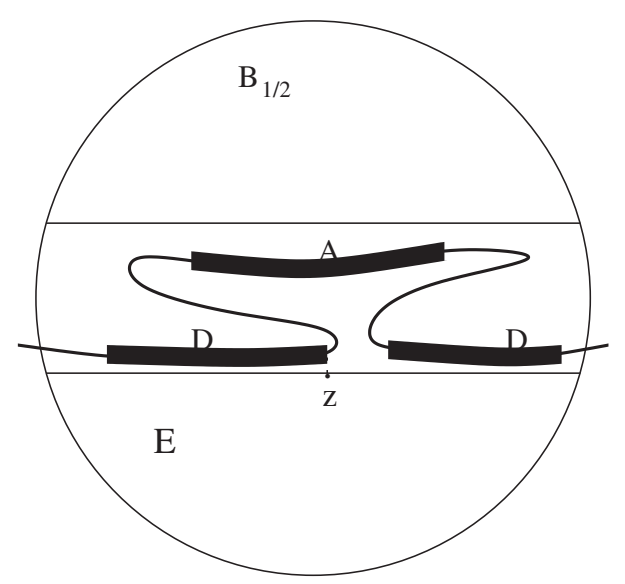

Figure 13. The contact sets $A$ and $D$

The sets $A$ and $D$ are disjoint since they lie on different sides of the plane $x_{n}=-\varepsilon / 2$. Moreover, they both project along $e_{n}$ in $B_{3 / 10}^{\prime}\left(z^{\prime}\right)$. We choose $\mu$ small universal such that

$$
\mathcal{H}^{n-1}\left(\pi_{n}(A)\right)+\mathcal{H}^{n-1}\left(\pi_{n}(D)\right) \geq \mathcal{H}^{n-1}\left(B_{3 / 10}^{\prime}\left(z^{\prime}\right)\right)+c_{0} / 2 .
$$

This implies that the projections $\pi_{n}(A)$ and $\pi_{n}(D)$ have an intersection of at least $c_{0} / 2$ measure. This gives

$$
P_{B_{1 / 2}}(E) \geq \mathcal{H}^{n-1}\left(B_{1 / 2}^{\prime}\right)+c_{0} / 3 \text {. }
$$

On the other hand, by comparing $E$ with $\left\{x_{n} \leq-\varepsilon\right\}$ in $B_{1 / 2}$, we find from the minimality of $E$ that

$$
P_{B_{1 / 2}} E \leq \mathcal{H}^{n-1}\left(B_{1 / 2}^{\prime}\right)+C \varepsilon,
$$

and we reach a contradiction if $\varepsilon$ is small.

\section{The flatness theorem for minimizers}

In the next three sections we prove the analogue of Theorem 5.2 for phase transitions, i.e. minimizers of the energy

$$
J(u, \Omega)=\int_{\Omega} \frac{1}{2}|\nabla u|^{2}+W(u) d x .
$$

The corresponding flatness theorem is the following.

THEOREM 7.1 (Improvement of flatness). Let $u$ be a minimizer of $J$ in the cylinder

$$
\left\{\left|x^{\prime}\right|<l\right\} \times\left\{\left|x_{n}\right|<l\right\}
$$

and assume that

$$
0 \in\{u=0\} \cap\left\{\left|x^{\prime}\right|<l\right\} \subset\left\{\left|x_{n}\right|<\theta\right\} .
$$




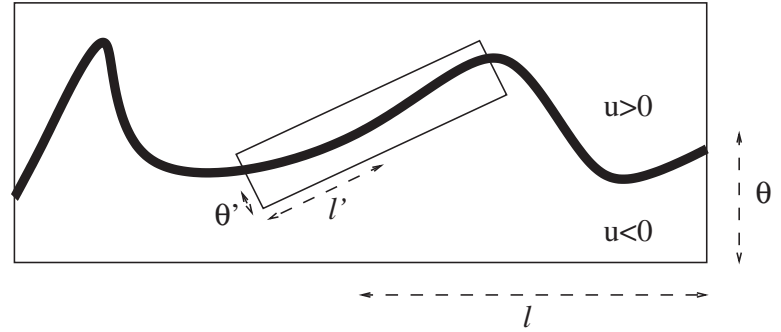

FiguRE 14. Improvement of flatness

Given $\theta_{0}>0$ there exists $\varepsilon_{1}\left(\theta_{0}\right)>0$ depending on $n, W$ and $\theta_{0}$ such that if

$$
\frac{\theta}{l} \leq \varepsilon_{1}\left(\theta_{0}\right), \quad \theta_{0} \leq \theta
$$

then

$$
\{u=0\} \cap\left\{\left|\pi_{\xi} x\right|<\eta_{2} l\right\} \subset\left\{|x \cdot \xi|<\eta_{1} \theta\right\},
$$

for some unit vector $\xi$, where $0<\eta_{1}<\eta_{2}<1$ are constants depending only on $n .\left(\pi_{\xi}\right.$ denotes the projection along $\xi$ )

The theorem is valid for any level set $s$ with $|s|<1$, but it was stated for $s=0$ for simplicity. As a consequence of this flatness theorem we obtain the following important corollary:

Corollary 7.2. Let $u$ be a global minimizer of $J$ in $\mathbb{R}^{n}$. Suppose that the 0 level set $\{u=0\}$ is asymptotically flat at $\infty$, i.e. there exist sequences of positive numbers $\theta_{k}, l_{k}$ and unit vectors $\xi_{k}$ with $l_{k} \rightarrow \infty, \theta_{k} l_{k}^{-1} \rightarrow 0$ such that

$$
\{u=0\} \cap B_{l_{k}} \subset\left\{\left|x \cdot \xi_{k}\right|<\theta_{k}\right\} .
$$

Then the 0 level set is a hyperplane and $u$ is one-dimensional.

By saying that $u$ is one-dimensional we understand that $u$ depends only on one direction $\xi$, i.e. $u=g(x \cdot \xi)$ for some function $g$. In our case, $g$ is a minimizer in 1D and can be computed explicitly from $W$. The function $g$ is unique up to translations.

Proof. Without loss of generality assume $u(0)=0$. Fix $\theta_{0}>0$, and choose $k$ large such that $\theta_{k} l_{k}^{-1} \leq \varepsilon \leq \varepsilon_{1}\left(\theta_{0}\right)$.

If $\theta_{k} \geq \theta_{0}$ then we apply Theorem 7.1 and obtain that $\{u=0\}$ is trapped in a flatter cylinder. We apply Theorem 7.1 repeatedly till the height of the cylinder becomes less than $\theta_{0}$.

In some system of coordinates we obtain

$$
\{u=0\} \cap\left(\left\{\left|y^{\prime}\right|<l_{k}^{\prime}\right\} \times\left\{\left|y_{n}\right|<l_{k}^{\prime}\right\}\right) \subset\left\{\left|y_{n}\right| \leq \theta_{k}^{\prime}\right\},
$$

with $\theta_{0} \geq \theta_{k}^{\prime} \geq \eta_{1} \theta_{0}$ and $\theta_{k}^{\prime} l_{k}^{-1} \leq \theta_{k} l_{k}^{-1} \leq \varepsilon$, hence

$$
l_{k}^{\prime} \geq \frac{\eta_{1}}{\varepsilon} \theta_{0} .
$$




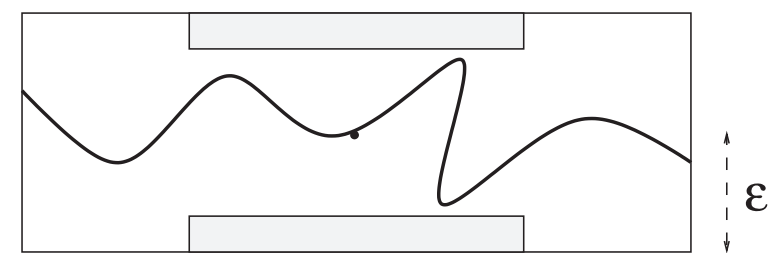

FiguRE 15. Harnack inequality

We let $\varepsilon \rightarrow 0$ and obtain that $\{u=0\}$ is included in an infinite strip of width $\theta_{0}$. We then let $\theta \rightarrow 0$ and obtain that $\{u=0\}$ is a hyperplane. Similarly, all the level sets are hyperplanes which implies that $u$ is onedimensional.

This corollary gives one of our main results: the uniqueness up to rotations of global minimizers in low dimensions (see Section 10).

The proof of Theorem 7.1 is quite involved. The idea is to reproduce the arguments from the minimal surface case given in the previous two sections. We present the proof of Theorem 7.1, with the exception of a number of technical lemmas that can be found in the original paper [55].

Again we will prove the improvement of flatness from a version of Harnack inequality.

THEOREM 7.3 (Harnack inequality for minimizers). Let $u$ be a minimizer of $J$ in the cylinder

$$
\left\{\left|x^{\prime}\right|<l\right\} \times\left\{\left|x_{n}\right|<l\right\}
$$

and assume that

$$
0 \in\{u=0\} \subset\left\{\left|x_{n}\right|<\theta\right\} .
$$

Given $\theta_{0}>0$ there exists $\varepsilon_{0}\left(\theta_{0}\right)>0$ depending on $n, W$ and $\theta_{0}$, such that if

$$
\theta l^{-1} \leq \varepsilon_{0}\left(\theta_{0}\right), \quad \theta_{0} \leq \theta,
$$

then

$$
\{u=0\} \cap\left\{\left|x^{\prime}\right|<\eta_{0} l\right\} \subset\left\{\left|x_{n}\right|<\left(1-\eta_{0}\right) \theta\right\},
$$

where $\eta_{0}>0$ is a small constant depending on $n$ and $W$.

In the remaining part of this section we show that Theorem 7.3 implies Theorem 7.1. We start by introducing some notation.

Notation. We denote points as follows

$$
\begin{gathered}
X=\left(x, x_{n+1}\right)=\left(x^{\prime}, x_{n}, x_{n+1}\right) \in \mathbb{R}^{n+1} \\
X \in \mathbb{R}^{n+1}, \quad x^{\prime} \in \mathbb{R}^{n-1}, \quad x \in \mathbb{R}^{n}, \quad\left|x_{n+1}\right|<1 .
\end{gathered}
$$


We use the following notation:

- $B(x, r)$ is the ball of center $x$ and radius $r$ in $\mathbb{R}^{n}$;

- $\mathcal{B}(X, r)$ is the ball of center $X$ and radius $r$ in $\mathbb{R}^{n+1}$;

- $\angle\left(\nu_{1}, \nu_{2}\right) \in(0, \pi)$ is the angle between the vectors $\nu_{1}$ and $\nu_{2}$;

- $\pi_{\nu} X=X-(X \cdot \nu) \nu$ is the projection along $\nu$;

- $P_{\nu}$ is the hyperplane perpendicular to $\nu$ going through the origin;

- $\pi_{i}:=\pi_{e_{i}}, P_{i}:=P_{e_{i}}$.

Constants depending on $n$, and $W$ are called universal and we denote them by $C, c, \bar{C}_{i}, \bar{c}_{i}$. We write $\bar{C}_{i}, \bar{c}_{i}$ for constants that we use throughout the paper and by $C, c$ various constants used in proofs that may change from line to line.

In the proof we often construct radial barriers

$$
x_{n+1}=g(|x|-r),
$$

from an increasing real function $g: I \rightarrow[-1,1]$ from some interval $I$ containing 0 . In order to check wether or not such a barrier is a subsolution or supersolution we have to check inequalities involving $g, g^{\prime}$ and $g^{\prime \prime}$. In this cases we consider $s=g$ as the new variable and we define a new function on $I$

$$
h(s):=\frac{1}{2}\left(\frac{d g}{d t}\right)^{2} .
$$

Straightforward computations give

$$
g^{\prime}=\sqrt{2 h}, \quad g^{\prime \prime}=h^{\prime}
$$

and now the inequalities involve only $h$ and $h^{\prime}$ in $I$. We can reconstruct $g$ from $h$ (up to a translation) since

$$
H(s):=\int_{0}^{s} \frac{1}{\sqrt{2 h(\zeta)}} d \zeta=g^{-1}(s)-g^{-1}(0) .
$$

In particular, if we define

$$
\begin{aligned}
h_{0}(s) & :=W(s) \\
H_{0}(s) & :=\int_{0}^{s} \frac{1}{\sqrt{2 h_{0}(\zeta)}} d \zeta
\end{aligned}
$$

and

$$
g_{0}(t):=H_{0}^{-1}(t)
$$

then we find

$$
g_{0}^{\prime \prime}(t)=h_{0}^{\prime}\left(g_{0}(t)\right)=W^{\prime}\left(g_{0}\right)
$$

thus, $g_{0}$ is the one dimensional solution of our semilinear equation.

First we show that the 0 level set of a minimizer satisfies in some sense an equation in the viscosity sense. 
Proposition 7.4. Let $u$ be a minimizer of $J$ in $\left\{\left|x^{\prime}\right|<l\right\} \times\left\{\left|x_{n}\right|<l\right\}$ and assume that $u(0)=0$ and $u<0$ below the graph of the quadratic polynomial

$$
\Gamma_{1}:=\left\{x_{n}=P_{1}\left(x^{\prime}\right)=\frac{\theta}{l^{2}} \frac{1}{2} x^{\prime T} M_{1} x^{\prime}+\frac{\theta}{l} \xi \cdot x^{\prime}\right\},
$$

where $M_{1}$ is a $(n-1) \times(n-1)$ symmetric matrix. Also assume

$$
\left\|M_{1}\right\|<\delta^{-1}, \quad|\xi|<\delta^{-1}
$$

for some small $\delta>0$. There exists $\sigma(\delta)>0$ small, such that if

$$
\theta l^{-1} \leq \sigma(\delta), \quad \theta \geq \delta
$$

then

$$
\operatorname{tr} M_{1} \leq \delta
$$

As a consequence of this proposition we end up with a version of the Theorem of Modica in the viscosity sense. This can be viewed as the analogue of Lemma 5.4 to the case of minimizers of $J$.

Proposition 7.5 (Modica theorem - viscosity sense). Let u be a minimizer of $J$ and assume that $u(0)=0$. Consider the graph of a quadratic polynomial

$$
\Gamma=\left\{x_{n}=P\left(x^{\prime}\right):=\frac{1}{2} x^{\prime T} M x^{\prime}\right\},
$$

that satisfies

$$
\triangle P=\operatorname{tr} M>\delta_{0}\|M\|, \quad\|M\| \leq \delta_{0}^{-1},
$$

for some $\delta_{0}>0$ small.

Then $\Gamma$ cannot touch by below $\left\{u_{\varepsilon}=0\right\}$ at 0 in a $\delta_{0}(\triangle P)^{-\frac{1}{2}} \varepsilon^{\frac{1}{2}}$ neighborhood, if $\varepsilon \leq \sigma_{0}\left(\delta_{0}\right)$ where $\sigma_{0}\left(\delta_{0}\right)>0$ depends on $\delta_{0}$ and the universal constants.

By " $\Gamma$ touches by below $\left\{u_{\varepsilon}=0\right\}$ at 0 in a $\delta_{0}(\triangle P)^{-\frac{1}{2}} \varepsilon^{\frac{1}{2}}$ neighborhood" we understand

$$
\left\{u_{\varepsilon}=0\right\} \cap\left\{x_{n}<P\left(x^{\prime}\right)\right\} \cap\left\{|x|<\delta_{0}(\triangle P)^{-\frac{1}{2}} \varepsilon^{\frac{1}{2}}\right\}=\emptyset .
$$

Proposition 7.5 says that $\left\{u_{\varepsilon}=0\right\}$ satisfies the mean curvature equation in some weak viscosity sense in which we have to specify the size of the neighborhood around the touching point. The size of the neighborhood depends on both the polynomial $P$ and $\varepsilon$.

If $P$ is fixed and $\varepsilon \rightarrow 0$ then the radius of the neighborhood converges to 0 . In particular, if $\left\{u_{\varepsilon}=0\right\}$ converges uniformly to a surface, then this surface satisfies in the viscosity sense the mean curvature equation.

One way to interpret the above proposition is the following:

Suppose that $P$ has positive mean curvature and let $\delta_{0}$ be small such that (7.1) holds. In order to obtain a contradiction that $P$ touches $\left\{u_{\varepsilon}=0\right\}$ 
by below at the origin we have to ensure that it stays below in a whole neighborhood. The size of this neighborhood is given by the first radius for which $P$ separates at just one point at a distance $\delta_{0} \varepsilon$ from $x_{n}=0$. Indeed, if $r$ denotes the radius of this neighborhood then,

$$
\|M\| r^{2} \geq 2 \delta_{0} \varepsilon \Rightarrow r^{2} \geq \delta_{0}\|M\|^{-1} \varepsilon \geq \delta_{0}^{2}(\triangle P)^{-1} \varepsilon .
$$

Hence, if $\varepsilon<\sigma_{0}\left(\delta_{0}\right)$ then $P$ cannot touch by below $\left\{u_{\varepsilon}=0\right\}$ at 0 in the $r$ neighborhood.

Next we show that Proposition 7.5 follows from Proposition 7.4.

Assume by contradiction that for some

$$
\varepsilon \leq \sigma_{0}\left(\delta_{0}\right):=\sigma^{2}\left(\frac{\delta_{0}^{2}}{4}\right)
$$

$\Gamma$ touches $\left\{u_{\varepsilon}=0\right\}$ by below at 0 in a $\delta_{0}(\Delta P)^{-\frac{1}{2}} \varepsilon^{\frac{1}{2}}$ neighborhood. By rescaling we find that

$$
\left\{x_{n}=\frac{\varepsilon}{2} x^{\prime T} M x^{\prime}\right\}
$$

touches by below $\{u=0\}$ at 0 in a $\delta_{0}(\triangle P)^{-\frac{1}{2}} \varepsilon^{-\frac{1}{2}}$ neighborhood.

We apply Lemma 7.4 with

$$
l=\frac{\delta_{0}}{2}(\operatorname{tr} M)^{-\frac{1}{2}} \varepsilon^{-\frac{1}{2}}, \quad \theta=\frac{\delta_{0}^{2}}{4}, \quad M_{1}=(\operatorname{tr} M)^{-1} M, \quad \xi=0
$$

and

$$
\delta:=\frac{\delta_{0}^{2}}{4}
$$

thus,

$$
\Gamma_{1}=\left\{x_{n}=\frac{\varepsilon}{2} x^{\prime T} M x^{\prime}\right\}
$$

Since

$$
\begin{aligned}
\left\|M_{1}\right\| & =(\operatorname{tr} M)^{-1}\|M\|<\delta_{0}^{-1}<\delta^{-1} \\
\theta l^{-1} & =\frac{\delta_{0}}{2}(\operatorname{tr} M)^{\frac{1}{2}} \varepsilon^{\frac{1}{2}}<\varepsilon^{\frac{1}{2}} \leq \sigma\left(\frac{\delta_{0}^{2}}{4}\right)=\sigma(\delta)
\end{aligned}
$$

we conclude

$$
\delta \geq \operatorname{tr} M_{1}=1
$$

which is a contradiction.

Next we show that Theorem 7.3 implies Theorem 7.1. The proof is by compactness and is similar to the one from minimal surfaces. Assume by contradiction that there exist $u_{k}, \theta_{k}, l_{k}, \xi_{k}$ such that $u_{k}$ is a local minimizer of $J, u_{k}(0)=0$, the level set $\left\{u_{k}=0\right\}$ stays in the flat cylinder

$$
\left\{\left|x^{\prime}\right|<l_{k}\right\} \times\left\{\left|x_{n}\right|<\theta_{k}\right\}
$$

$\theta \geq \theta_{0}, \theta_{k} l_{k}^{-1} \rightarrow 0$ as $k \rightarrow \infty$ for which the conclusion of Theorem 7.1 doesn't hold. 
Let $A_{k}$ be the rescaling of the 0 level sets given by

$$
\begin{gathered}
\left(x^{\prime}, x_{n}\right) \in\left\{u_{k}=0\right\} \mapsto\left(y^{\prime}, y_{n}\right) \in A_{k} \\
y^{\prime}=x^{\prime} l_{k}^{-1}, \quad y_{n}=x_{n} \theta_{k}^{-1} .
\end{gathered}
$$

Claim 1. $A_{k}$ has a subsequence that converges uniformly on $\left|y^{\prime}\right| \leq 1 / 2$ to a set $A_{\infty}=\left\{\left(y^{\prime}, w\left(y^{\prime}\right)\right), \quad\left|y^{\prime}\right| \leq 1 / 2\right\}$ where $w$ is a Holder continuous function. In other words, given $\varepsilon$, all but a finite number of the $A_{k}$ 's from the subsequence are in an $\varepsilon$ neighborhood of $A_{\infty}$.

Proof. Fix $y_{0}^{\prime},\left|y_{0}^{\prime}\right| \leq 1 / 2$ and suppose $\left(y_{0}^{\prime}, y_{k}\right) \in A_{k}$. We apply Theorem 7.3 for the function $u_{k}$ in the cylinder

$$
\left\{\left|x^{\prime}-l_{k} y_{0}^{\prime}\right|<l_{k} / 2\right\} \times\left\{\left|x_{n}-\theta_{k} y_{k}\right|<2 \theta_{k}\right\}
$$

in which the set $\left\{u_{k}=0\right\}$ is trapped. Thus, there exist a universal constant $\eta_{0}>0$ and an increasing function $\varepsilon_{0}(\theta)>0, \varepsilon_{0}(\theta) \rightarrow 0$ as $\theta \rightarrow 0$, such that $\left\{u_{k}=0\right\}$ is trapped in the cylinder

$$
\left\{\left|x^{\prime}-l_{k} y_{0}\right|<\eta_{0} l_{k} / 2\right\} \times\left\{\left|x_{n}-\theta_{k} y_{k}\right|<2\left(1-\eta_{0}\right) \theta_{k}\right\}
$$

provided that $4 \theta_{k} l_{k}^{-1} \leq \varepsilon_{0}\left(2 \theta_{k}\right)$. Rescaling back we find that

$$
A_{k} \cap\left\{\left|y^{\prime}-y_{0}^{\prime}\right| \leq \eta_{0} / 2\right\} \subset\left\{\left|y_{n}-y_{k}\right| \leq 2\left(1-\eta_{0}\right)\right\} .
$$

We apply the Harnack inequality repeatedly and we find that

$$
A_{k} \cap\left\{\left|y^{\prime}-y_{0}^{\prime}\right| \leq \eta_{0}^{m} / 2\right\} \subset\left\{\left|y_{n}-y_{k}\right| \leq 2\left(1-\eta_{0}\right)^{m}\right\}
$$

provided that

$$
4 \theta_{k} l_{k}^{-1} \leq \eta_{0}^{m-1} \varepsilon_{0}\left(2\left(1-\eta_{0}\right)^{m} \theta_{k}\right)
$$

Since these inequalities are satisfied for all $k$ large we conclude that (7.2) holds for all but a finite number of $k$ 's.

There exist positive constants $\alpha, \beta$ depending only on $\eta_{0}$, such that if (7.2) holds for all $m \leq m_{0}$ then $A_{k}$ is above the graph

$$
y_{n}=y_{k}-2\left(1-\eta_{0}\right)^{m_{0}-1}-\alpha\left|y^{\prime}-y_{0}^{\prime}\right|^{\beta}
$$

in the cylinder $\left|y^{\prime}\right| \leq 1 / 2$.

Taking the supremum over these functions as $y_{0}^{\prime}$ varies we obtain that $A_{k}$ is above the graph of a Hölder function $y_{n}=a_{k}\left(y^{\prime}\right)$. Similarly we obtain that $A_{k}$ is below the graph of a Hölder function $y_{n}=b_{k}\left(y^{\prime}\right)$. Notice that

$$
b_{k}-a_{k} \leq 4\left(1-\eta_{0}\right)^{m_{0}-1}
$$

and that $a_{k}, b_{k}$ have a modulus of continuity bounded by the Hölder function $\alpha t^{\beta}$. From Arzela-Ascoli Theorem we find that there exists a subsequence 
$a_{k_{p}}$ which converges uniformly to a function $w$. Using (7.3) we obtain that $b_{k_{p}}$, and therefore $A_{k_{p}}$, converge uniformly to $w$.

Claim 2. The function $w$ is harmonic (in the viscosity sense).

Proof. The proof is by contradiction. Fix a quadratic polynomial

$$
y_{n}=P\left(y^{\prime}\right)=\frac{1}{2} y^{\prime T} M y^{\prime}+\xi \cdot y^{\prime}, \quad\|M\|<\delta^{-1}, \quad|\xi|<\delta^{-1}
$$

such that $\triangle P>\delta, P\left(y^{\prime}\right)+\delta\left|y^{\prime}\right|^{2}$ touches the graph of $w$, say, at 0 for simplicity, and stays below $w$ in $\left|y^{\prime}\right|<2 \delta$. Thus, for all $k$ large we find points $\left(y_{k}{ }^{\prime}, y_{k_{n}}\right)$ close to 0 such that $P\left(y^{\prime}\right)+$ const touches $A_{k}$ by below at $\left(y_{k}{ }^{\prime}, y_{k_{n}}\right)$ and stays below it in $\left|y^{\prime}-y_{k}{ }^{\prime}\right|<\delta$.

This implies that, after eventually a translation, there exists a surface

$$
\left\{x_{n}=\frac{\theta_{k}}{l_{k}^{2}} \frac{1}{2} x^{\prime T} M x^{\prime}+\frac{\theta_{k}}{l_{k}} \xi_{k} \cdot x^{\prime}\right\}, \quad\left|\xi_{k}\right|<2 \delta^{-1}
$$

that touches $\left\{u_{k}=0\right\}$ at the origin and stays below it in the cylinder $\left|x^{\prime}\right|<\delta l_{k}$. We write the above surface in the form

$$
\left\{x_{n}=\frac{\delta^{2} \theta_{k}}{2\left(\delta l_{k}\right)^{2}} x^{\prime T} M x^{\prime}+\frac{\delta^{2} \theta_{k}}{\delta l_{k}} \delta^{-1} \xi_{k} \cdot x^{\prime}\right\},
$$

and we contradict Proposition 7.4 since $\theta_{k} \geq \theta_{0}, \theta_{k} l_{k}^{-1} \rightarrow 0$ and $\triangle P>\delta$.

Since $w$ is harmonic, there exist $0<\eta_{1}<\eta_{2}$ small (depending only on n) such that

$$
\left|w-\xi \cdot y^{\prime}\right|<\eta_{1} / 2 \text { for }\left|y^{\prime}\right|<2 \eta_{2} \text {. }
$$

Rescaling back and using the fact that $A_{k}$ converge uniformly to the graph of $w$ we conclude that for $k$ large enough

$$
\left\{u_{k}=0\right\} \cap\left\{\left|x^{\prime}\right|<3 l_{k} \eta_{2} / 2\right\} \subset\left\{\left|x_{n}-\theta_{k} l_{k}^{-1} \xi \cdot x^{\prime}\right|<3 \theta_{k} \eta_{1} / 4\right\} .
$$

This is a contradiction with the fact that $u_{k}$ doesn't satisfy the conclusion of the Theorem 7.1 .

\section{A family of sliding surfaces}

We prove Theorem 7.3 following the strategy of Section 5. The key step is to obtain estimates in measure. Since the level sets do not satisfy any equation on their own, one needs to look at their full collection.

In this section we introduce a family of sliding surfaces. These are rotation surfaces in $\mathbb{R}^{n+1}$ which we denote by $\mathcal{S}(Y, R)$. We say that the point $Y$ is the center of $\mathcal{S}$ and $R$ the radius. These surfaces are perturbations of the one dimensional solution. Roughly speaking they are obtained by first rotating $g_{0}(t)$ around the axis $t=-R$, and then modifying it outside the $s$ level sets with $|s|<1 / 2$, so that the resulting surface is a supersolution in the set $\left\{\left|x_{n+1}\right| \geq 1 / 2\right\}$. 
The surfaces $\mathcal{S}$ in our setting play the same role as the paraboloids in Section 5. The key property that $\mathcal{S}$ satisfies is the following: Suppose that for fixed $R$, some surfaces $\mathcal{S}(Y, R)$ are tangent by above to the graph of $u$. Then the contact points project along $e_{n}$ into a set with measure comparable with the measure of the projections of the centers $Y$ along $e_{n}$ (see Proposition 8.1).

We proceed with the explicit construction of $\mathcal{S}$.

For $\left|y_{n+1}\right| \leq 1 / 4$, we define $\mathcal{S}(Y, R)$ as

$$
\mathcal{S}(Y, R):=\left\{x_{n+1}=g_{y_{n+1}, R}\left(H_{0}\left(y_{n+1}\right)+|x-y|-R\right)\right\},
$$

where the function $g_{s_{0}, R}$, respectively $h_{s_{0}, R}, H_{s_{0}, R}$ associated with it, are constructed below for $\left|s_{0}\right| \leq 1 / 4$ and large $R$. For simplicity of notation we denote them by $g, h, H$.

Denote

$$
\bar{C}=1+8(n-1) \max \sqrt{W}
$$

and let $\varphi$ be such that

$$
\frac{1}{\sqrt{2 \varphi(s)}}=\frac{1}{\sqrt{2 h_{0}(s)}}-\frac{\bar{C}_{0}}{R}\left(s-s_{0}\right),
$$

where $\bar{C}_{0}$ is large enough so that the following holds

$$
\begin{array}{ll}
\varphi(s)<h_{0}(s)-2 \bar{C} R^{-1}, & \text { if } s \in[-3 / 4,-1 / 2] \\
\varphi(s)>h_{0}(s)+2 \bar{C} R^{-1}, & \text { if } s \in[1 / 2,3 / 4] .
\end{array}
$$

Let $s_{R}$ near -1 be such that $h_{0}\left(s_{R}\right)=R^{-1}$, hence $1+s_{R} \sim R^{-\frac{1}{2}}$. We define $h_{s_{0}, R}:\left[s_{R}, 1\right] \rightarrow \mathbb{R}$ as

$$
h(s)=\left\{\begin{array}{l}
h_{0}(s)-h_{0}\left(s_{R}\right)-\bar{C} R^{-1}\left(s-s_{R}\right) \quad \text { if } s \in\left[s_{R},-\frac{1}{2}\right] \\
\varphi(s) \quad \text { if } s \in(-1 / 2,1 / 2) \\
h_{0}(s)+R^{-1}+\bar{C} R^{-1}(1-s) \quad \text { if } s \in\left[\frac{1}{2}, 1\right] .
\end{array}\right.
$$

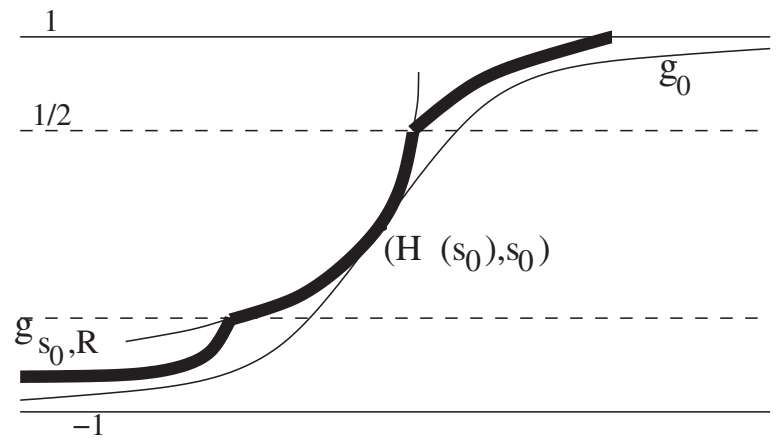

Figure 16. The profile of $\mathcal{S}(Y, R)$ 
For $R$ large, $h(s) \geq c(1+s)\left(s-s_{R}\right)$ on $\left[s_{R}, 0\right]$, thus $h$ is positive on $\left(s_{R}, 1\right]$. Define

$$
H_{s_{0}, R}(s)=H_{0}\left(s_{0}\right)+\int_{s_{0}}^{s} \frac{1}{\sqrt{2 h(\zeta)}} d \zeta
$$

and for $R$ large enough

$$
\begin{gathered}
H\left(s_{R}\right) \geq H_{0}\left(s_{0}\right)-\int_{s_{R}}^{s_{0}} \frac{1}{\sqrt{c(1+\zeta)\left(\zeta-s_{R}\right)}} d \zeta \geq-C \log R \\
H(1) \leq H_{0}\left(s_{0}\right)+\int_{s_{0}}^{1} \frac{1}{\sqrt{c(1-\zeta)^{2}+R^{-1}}} d \zeta \leq C \log R .
\end{gathered}
$$

Finally we define $g_{s_{0}, R}$ as

$$
g_{s_{0}, R}(t)=\begin{array}{ll}
s_{R} & \text { if } t<H\left(s_{R}\right) \\
H^{-1}(t) & \text { if } H\left(s_{R}\right) \leq t \leq H(1)
\end{array}
$$

Now we list some properties of the surfaces $\mathcal{S}(Y, R)$ (see Figure 16).

1) We have

$$
\begin{array}{ll}
h(s)>h_{0}(s)-2 \bar{C} R^{-1}>\varphi(s), & \text { if } s \in[-3 / 4,-1 / 2], \\
h(s)<h_{0}(s)+2 \bar{C} R^{-1}<\varphi(s), & \text { if } s \in[1 / 2,3 / 4]
\end{array}
$$

and

$$
\begin{aligned}
& H(s)=H_{0}(s)-\frac{\bar{C}_{0}}{2 R}\left(s-s_{0}\right)^{2}, \quad \text { if }|s| \leq 1 / 2, \\
& H(s)>H_{0}(s)-\frac{\bar{C}_{0}}{2 R}\left(s-s_{0}\right)^{2} \quad \text { if } 1 / 2<|s|<3 / 4 .
\end{aligned}
$$

Let $\rho_{s_{0}, R}$ be the function whose graph is obtained from the graph of $g_{0}$ by the transformation

$$
(t, s) \mapsto\left(t-\frac{\bar{C}_{0}}{2 R}\left(s-s_{0}\right)^{2}, s\right) \quad \text { for }|s|<3 / 4 .
$$

From the formulas above we obtain that $g=\rho$ for $|s| \leq 1 / 2$, and $g<\rho$ at all other points where $\rho$ is defined. In other words, if $\mathbf{S}(Y, R)$ is the rotation surface

$$
\mathbf{S}(Y, R):=\left\{x_{n+1}=\rho_{y_{n+1}, R}\left(H_{0}\left(y_{n+1}\right)+|x-y|-R\right)\right\},
$$

then, $\mathcal{S}(Y, R)$ coincides with $\mathbf{S}(Y, R)$ in the set $\left|x_{n+1}\right| \leq 1 / 2$ and stays below it at all the other points where $\mathbf{S}$ is defined.

Notice that

$$
\mathbf{S}(Y, R) \subset\left\{\left|x_{n+1}\right| \leq 3 / 4\right\}
$$

and it is defined only in a neighborhood of the sphere $|x-y|=R$ which is the $y_{n+1}$ level set of $\mathbf{S}(Y, R)$. 
2) We remark that $\mathcal{S}(Y, R)$ is constant $s_{R}$ when

$$
|x-y| \leq R-C \log R,
$$

and grows from $s_{R}$ to 1 when

$$
R-C \log R \leq|x-y| \leq R+C \log R .
$$

3) If $s \in\left(s_{R},-1 / 2\right) \cup(1 / 2,1)$, then on the $s$ level set of $\mathcal{S}$

$$
\begin{aligned}
\triangle \mathcal{S} & \leq h^{\prime}(s)+2(n-1) R^{-1} \sqrt{2 h(s)} \\
& \leq h_{0}^{\prime}(s)-\bar{C} R^{-1}+4(n-1) R^{-1} \sqrt{h(s)}<h_{0}^{\prime}(s),
\end{aligned}
$$

hence, $\mathcal{S}$ is a supersolution on its $s$ level set. Moreover,

$$
\lim _{s \rightarrow \pm 1 / 2^{-}} H^{\prime}(s)<\lim _{s \rightarrow \pm 1 / 2^{+}} H^{\prime}(s), \quad \lim _{s \rightarrow 1^{-}} H^{\prime}(s)<\infty
$$

thus $\mathcal{S}(Y, R)$ is a strict supersolution for $\left|x_{n+1}\right| \geq 1 / 2$, that is $\mathcal{S}(Y, R)$ cannot touch by above the graph of a $C^{2}$ subsolution at a point $X$ with $\left|x_{n+1}\right| \geq 1 / 2$.

4) If $|s|<3 / 4$, then

$$
\left|\varphi^{\prime}(s)-h_{0}^{\prime}(s)\right| \leq C R^{-1}
$$

If $v_{S}$ denotes the function with graph $\mathbf{S}(Y, R)$ defined above, one has

$$
\left|\triangle v_{S}-h_{0}^{\prime}\left(v_{S}\right)\right| \leq C\left|\varphi^{\prime}-h_{0}^{\prime}\right|+C R^{-1} \leq C R^{-1}
$$

hence $v_{S}$ is an approximate solution of the equation with a $R^{-1}$ error.

Next proposition is quite surprising and is the key tool in proving Theorem 7.3. It is the analogue of the ABP estimate (Lemma 6.3) of Section 5.

Recall the notation $P_{n}:=\left\{x_{n}=0\right\}$.

Proposition 8.1 (Measure estimate for contact points). Let u be a $C^{2}$ subsolution i.e.

$$
\triangle u \geq h_{0}^{\prime}(u), \quad|u|<1
$$

Let $A$ be a closed set in

$$
P_{n} \cap\left\{\left|x_{n+1}\right| \leq 1 / 4\right\} .
$$

Assume that for each $Y \in A$ the surface $\mathcal{S}\left(Y+t_{n}, R\right), R$ large, stays above the graph of $u$ when $t \rightarrow-\infty$ and, as $t$ increases, it touches the graph by above for the first time at a point (contact point). If $B$ denotes the projection of the contact points along $e_{n}$ in $P_{n}$, then,

$$
\bar{\mu}_{0}|A| \leq|B|
$$

where $\bar{\mu}_{0}>0$ universal, small and $|A|$ represents the $n$-dimensional Lebesgue measure. 
Proof. Assume that $\mathcal{S}(Y, R)$ touches $u$ by above at the point $X=$ $(x, u(x))$. From the discussion above we find $|u(x)|<1 / 2$.

Denote by $\nu$ the normal to the surface at $X$, i.e.

$$
\nu=\left(\nu^{\prime}, \nu_{n+1}\right)=\frac{1}{\sqrt{1+|\nabla u|^{2}}}(-\nabla u, 1) .
$$

For any contact point $X$ the corresponding center $Y$ is given by

$$
Y(X)=\left(x+\frac{\nu^{\prime}}{\left|\nu^{\prime}\right|} \sigma, x_{n+1}+\omega\right)=F(X, \nu),
$$

where

$$
\begin{gathered}
\omega=R \bar{C}_{0}^{-1}\left(\nu_{n+1}\left|\nu^{\prime}\right|^{-1}-H_{0}^{\prime}\left(x_{n+1}\right)\right) \\
\sigma=-\frac{\bar{C}_{0}}{2 R} \omega^{2}+H_{0}\left(x_{n+1}\right)-H_{0}\left(x_{n+1}+\omega\right)+R .
\end{gathered}
$$

The function $F$ is smooth defined on

$$
\left\{X \in \mathbb{R}^{n+1}:\left|x_{n+1}\right|<1 / 2\right\} \times\left\{\nu \in \mathbb{R}^{n+1}:|\nu|=1, c_{1}<\nu_{n+1}<1-c_{1}\right\} .
$$

The differential $D_{X} Y$ is a linear map defined on $T_{X}$, the tangent plane at $X$, and

$$
D_{X} Y=F_{X}(X, \nu)+F_{\nu}(X, \nu) D_{X} \nu=F_{X}(X, \nu)-F_{\nu}(X, \nu) I I_{u}
$$

where $I I_{u}$ represents the second fundamental form of $u$ at $X$. Writing the formula above for the surface $\mathbf{S}(Y, R)$ at $X$, we find

$$
0=F_{X}(X, \nu)-F_{\nu}(X, \nu) I I_{S}
$$

thus,

$$
D_{X} Y=F_{\nu}(X, \nu)\left(I I_{S}-I I_{u}\right) .
$$

From (8.2) and (8.2), it is easy to check that

$$
\left\|F_{\nu}(X, \nu)\right\| \leq C R \text {. }
$$

Since $\mathbf{S}$ touches $u$ by above at $X$, we find that

$$
D^{2} v_{S}(x)-D^{2} u(x) \geq 0,
$$

where $v_{S}$ is the function whose graph is $\mathbf{S}$. On the other hand, from (8.2),

$$
\triangle v_{S} \leq h_{0}^{\prime}\left(x_{n+1}\right)+C R^{-1}=\triangle u+C R^{-1}
$$

which implies

$$
\left\|D^{2} S(x)-D^{2} u(x)\right\| \leq C R^{-1}
$$

or

$$
\left\|I I_{S}-I I_{u}\right\| \leq C R^{-1} .
$$

This gives

$$
\left\|D_{X} Y\right\| \leq C .
$$


The centers $Z$ for which $X \in \mathbf{S}(Z, R)$ describe a rotation surface, around $X$. Note if $\mathbf{S}(\cdot, R)$ is above $u$, then its center is above this surface. The normal to the surface at $Y(X)$, which we denote by $\tau$, belongs to the plane spanned by $\nu$ and $e_{n+1}$, and $c_{2}<\tau_{n+1}<1-c_{2}$. Thus, we have

$$
\left|\tau \cdot e_{n}\right| \leq C\left|\nu \cdot e_{n}\right|
$$

(notice that the tangent plane to the surface at $Y(X)$ is the range of $F_{\nu}(X, \nu)$.)

Let $\tilde{B}$ be the set of contact points, $\tilde{A}$ the set of the corresponding centers, $B=\pi_{n} \tilde{B}$ and $A=\pi_{n} \tilde{A}$. Remark that $\pi_{n}$ is injective on $\tilde{A}$ and $\tilde{B}$ by construction. From above, we know that $\tilde{A}$ belongs to a Lipschitz surface. One has

$$
\begin{aligned}
|A| & =\int_{\tilde{A}}\left|\tau(Y) \cdot e_{n}\right| d Y \leq \int_{\tilde{B}}\left|\tau(Y) \cdot e_{n}\right|\left|D_{X} Y\right| d X \\
& \leq C \int_{\tilde{B}}\left|\nu(X) \cdot e_{n}\right| d X=C|B|,
\end{aligned}
$$

which is the desired claim.

\section{Proof of Theorem 7.3}

In this section we prove Harnack inequality for minimizers of $J$. The main step is to show that if the 0 level set is close to the bottom $\left\{x_{n}=-\theta\right\}$ at one point, then the minimizer $u$ is close to the one-dimensional solution $g_{0}\left(x_{n}+\theta\right)$ in a set of large measure. This set is obtained as the contact set between surfaces $\mathcal{S}(Y, R)$ and the graph of $u$. The measure estimate for the contact set comes from Proposition 8.1.

We estimate the size of the contact set by the Lebesgue measure of its projection along $e_{n}$. It is often convenient to think of the graph of $u$ as a possibly multivalued graph over the set $P_{n}=\left\{x_{n}=0\right\}$. Since the contact points occur only in the strip $\left\{\left|x_{n+1}\right|<1 / 2\right\}$, we introduce the sets $L$ and $Q_{l} \subset L$

$$
\begin{aligned}
L & =P_{n} \cap\left\{\left|x_{n+1}\right| \leq 1 / 2\right\}, \\
Q_{l} & =L \cap\left\{\left|x^{\prime}\right| \leq l\right\} .
\end{aligned}
$$

Let us suppose we have a minimizer of $J$ defined in a cylinder of size $2 l$,

$$
\left\{\left|x^{\prime}\right|<2 l\right\} \times\left\{\left|x_{n}\right|<2 l\right\}
$$

such that the 0 level set stays above $\left\{x_{n}=0\right\}$ but close to it at one point above the origin

$$
\begin{gathered}
\{u=0\} \cap\left\{\left|x^{\prime}\right|<2 l\right\} \subset\left\{x_{n}>0\right\}, \\
(0, \theta) \in\{u=0\}, \quad \theta \geq \theta_{0},
\end{gathered}
$$

for some fixed $\theta_{0}$. We denote

$$
\varepsilon:=\frac{\theta}{l}
$$


Then the following result holds.

Proposition 9.1. There exist universal constants $\bar{C}_{*}, \bar{\mu}, \bar{c}_{1}$ such that if

$$
\bar{C}_{*}^{k} \varepsilon \leq \bar{c}_{1}, \quad l \geq C\left(\theta_{0}\right)
$$

then the set of points

$$
(x, u(x)) \in\left\{\left|x^{\prime}\right| \leq l\right\} \times\left\{\left|x_{n+1}\right| \leq 1 / 2\right\}
$$

that satisfy

$$
x_{n} \leq \bar{C}_{*}^{k} \theta+H_{0}(u(x))
$$

projects along $e_{n}$ into a set of measure greater than $\left(1-(1-\bar{\mu})^{k}\right)\left|Q_{l}\right|$.

The inequality above says that the point $(x, u(x))$ stays at distance less than $\bar{C}_{*}^{k} \theta$ in the $e_{n}$ direction from graph of the one dimensional solution $g_{0}\left(x_{n}\right)$.

This proposition is the analogue of Proposition 6.1 for minimal surfaces. The proof involves a number of technical lemmas that we describe below. We refer the reader to the original paper [55] for their proofs.

Since $(0, \theta)$ is close enough to the plane $\left\{x_{n}=0\right\}$ we expect to find interior contact points with the sliding surfaces $\mathcal{S}(Y, R)$ for appropriate centers $Y$ and radius $R$. This is explained in the next lemma. We remark that this step is straightforward in the case of minimal surfaces. The case of minimizers is more delicate since the bound on the 0 level does not guarantee that the contact points cannot occur on the boundary of the cylinder.

LEMMA 9.2 (Initial contact set). If $\varepsilon \leq \bar{c}_{2}$, then the points $(x, u(x))$ with the following properties

1) $\left|x^{\prime}\right|<l,|u(x)|<1 / 2$

2) there is a surface $\mathcal{S}\left(Y, R_{0}\right)$ that stays above $u$ in the cylinder $\left|x^{\prime}\right|<$ $l$ and touches its graph at $(x, u(x))$, where

$$
R_{0}=\frac{l^{2}}{\theta}, \quad l>\bar{C}_{1} \log R_{0}
$$

3) $\angle\left(\nu(x), \xi_{0}\right) \leq l R_{0}^{-1}$

4) $x_{n} \leq(1+b) \theta+H_{0}(u(x)), \quad b<1$ is a positive fixed number project along $e_{n}$ into a set of measure greater than $\bar{c}_{2}(l \sqrt{b})^{n-1}$ provided that $l \geq C\left(b, \theta_{0}\right)$.

Let $\tilde{D}_{k}$ represent the set of points on the graph of $u$ that have by above a tangent surface $\mathcal{S}\left(Y, R C^{-k}\right)$, where $C$ is a large universal constant. Suppose that we have control on the $e_{n}$ coordinate of these sets and denote by $D_{k}=$ $\pi_{n}\left(\tilde{D}_{k}\right)$ their projections along $e_{n}$ into $L$.

Recall that $\mathcal{S}\left(Y, R C^{-k}\right)$ is an approximate solution of our equation with a $C^{k} R^{-1}$ error. If $\mathcal{S}\left(Y, R C^{-k}\right)$ touches $u$ by above at $X_{0}$ then, from the classical Harnack inequality for uniformly elliptic equations, the two surfaces 
stay $C^{k} R^{-1}$ close to each other in a neighborhood of $X_{0}$ (see Lemma 9.3). Thus, if we denote

$$
E_{k}=\left\{Z \in L / \quad \operatorname{dist}\left(Z, D_{k}\right) \leq C_{1}\right\},
$$

then we control the $e_{n}$ coordinate of a set on the graph of $u$ that projects along $e_{n}$ into $E_{k}$. Proposition 9.1 states that, in measure, $E_{k}$ almost covers $Q_{l}$ as $k$ becomes larger and larger.

Looking at the graph of a solution between $-1 / 2$ and $1 / 2$ we see two different behaviors depending on the size of the neighborhood on which we focus our attention. Up to unit scale we see a Laplace equation and this is the reason why near a contact point the solution and the sliding surface stay very close to each other (see Lemma 9.3). At larger scales, the graph looks more like a minimal surface and the contact set behaves as in the minimal surface case. Lemma 9.4 below says that near (large scale) a point $Z \in D_{k}$ we can find a set of positive measure in $D_{k+1}$. Using a covering argument we show that the sets $E_{k}$ "almost" cover $Q_{l}$ as $k$ increases.

Next we state the two lemmas that describe the different behaviors. We use the following notation:

$$
\nu(x):=\frac{\nabla u}{|\nabla u|}(x) \in \mathbb{R}^{n} .
$$

We assume that there exists a surface $\mathcal{S}\left(Y_{0}, R\right)$ that touches the graph of a solution $u$ by above at a point $X_{0}=\left(x_{0}, u\left(x_{0}\right)\right)$ with

$$
\angle\left(\nu\left(x_{0}\right), e_{n}\right) \leq \pi / 4 \text {. }
$$

LEMma 9.3 (Small scale measure estimate). Given a constant $a>1$ large, there exists $C(a)>0$ depending also on a such that for each point $Z \in L \cap \mathcal{B}\left(\pi_{n} X_{0}, a\right)$ there exists $x$ with

1) $\pi_{n}(x, u(x))=Z, \quad\left|x-x_{0}\right| \leq 2 a$,

2) $\left(x-x_{0}\right) \cdot \nu\left(x_{0}\right) \leq H_{0}(u(x))-H_{0}\left(u\left(x_{0}\right)\right)+C(a) R^{-1}$.

Next lemma corresponds to Lemma 6.3 in the minimal surface case.

LEMMA 9.4 (Large scale measure estimate). Suppose that $u$ is defined in the cylinder $\left\{\left|x^{\prime}\right|<l\right\} \times\left\{\left|x_{n}\right|<l\right\}$ and satisfies the hypothesis above with

$$
\left|x_{0 n}\right|<l / 4, \quad\left|x_{0}^{\prime}\right|=q, \quad q<l / 4 .
$$

There exist constants $\bar{C}_{1}, \bar{C}_{2}$, such that if

$$
q \geq \bar{C}_{1}, \quad R \geq l \bar{C}_{1}, \quad l \geq \bar{C}_{1} \log R
$$

then the set of points $(x, u(x))$ with the following four properties

1) $\left|x^{\prime}\right|<q / 15,\left|x-x_{0}\right|<2 q,|u(x)|<1 / 2$,

2) there is a surface $\mathcal{S}\left(Y, R / \bar{C}_{2}\right)$ that stays above $u$ and touches its graph at $(x, u(x))$,

3) $\angle\left(\nu(x), \nu\left(x_{0}\right)\right) \leq \bar{C}_{1} q R^{-1}$, 
4) $\left(x-x_{0}\right) \cdot \nu\left(x_{0}\right) \leq \bar{C}_{1} q^{2} R^{-1}+H_{0}(u(x))-H_{0}\left(u\left(x_{0}\right)\right)$, projects along $e_{n}$ into a set of measure greater than $q^{n-1} / \bar{C}_{1}$.

REMARK. The term $H_{0}(u(x))-H_{0}\left(u\left(x_{0}\right)\right)$ that appears in property 2 of Lemma 9.3 and property 4 of Lemma 9.4 represents the distance between the $u(x)$ level surface and the $u\left(x_{0}\right)$ level surface of a one dimensional solution.

Now we state the covering lemma that links the two scales.

Lemma 9.5 (Covering lemma). Let $D_{k}$ be closed sets, $D_{k} \subset L$, with the following properties:

1) $D_{0} \cap Q_{l} \neq \emptyset, \quad D_{0} \subset D_{1} \subset D_{2} \ldots$

2) if $Z_{0} \in D_{k} \cap Q_{2 l}, Z_{1} \in L,\left|Z_{1}-Z_{0}\right|=q$ and $2 l \geq q \geq a$ then,

$$
\left|D_{k+1} \cap \mathcal{B}\left(Z_{1}, q / 10\right)\right| \geq \mu_{1}\left|\mathcal{B}\left(Z_{1}, q\right) \cap L\right|
$$

where $a>1$ (large), $\mu_{1}$ (small) are given positive constants and $l>2 a$.

Denote by $E_{k}$ the set

$$
E_{k}:=\left\{Z \in L / \quad \operatorname{dist}\left(Z, D_{k}\right) \leq a\right\}
$$

Then there exists $\mu>0$ depending on $n, \mu_{1}$ such that

$$
\left|Q_{l} \backslash E_{k}\right| \leq(1-\mu)^{k}\left|Q_{l}\right|
$$

The proof of Proposition 9.1 follows from the lemmas above by showing that the sets $D_{k}$ and $E_{k}$ satisfy the hypotheses of the covering lemma above. The initial set $D_{0}$ is given by Lemma 9.2.

Proof of TheOrem 7.3. We assume that $u$ is a local minimizer of $J$ in the cylinder

$$
\left\{\left|x^{\prime}\right|<2 l\right\} \times\left\{\left|x_{n}\right|<2 l\right\}
$$

and

$$
u>0 \quad \text { if } x_{n}>\theta, \quad u<0 \quad \text { if } x_{n}<-\theta, \quad u(0)=0 .
$$

We show that if the 0 level set is close to $x_{n}=-\theta$ at a point in $\left|x^{\prime}\right|<l / 4$ then the energy of $u$ is large and obtain a contradiction.

First we apply Lemma 9.2 upside-down and obtain that there exists a small universal constant $c_{1}$ such that the points $(x, u(x))$ with

$$
x_{n} \geq-\frac{\theta}{4}+H_{0}(u(x)), \quad\left|x^{\prime}\right| \leq \frac{l}{2}, \quad|u(x)| \leq \frac{1}{2}
$$

project along $e_{n}$ in a set of measure greater than $c_{1} l^{n-1}$, provided that $\theta l^{-1} \leq \varepsilon_{2}\left(\theta_{0}\right)$ is small. 
On the other hand, from Proposition 9.1 we find universal constants $\bar{C}_{*}$, $\bar{\mu}$ such that if

$$
\begin{gathered}
\{u=0\} \cap\left\{\left|x^{\prime}\right|<l / 4\right\} \cap\left\{x_{n}<\left(-1+\bar{C}_{*}^{-k_{0}} / 4\right) \theta\right\} \neq \emptyset, \\
\theta l^{-1} \leq \varepsilon_{3}\left(\theta_{0}, k_{0}\right)
\end{gathered}
$$

then the set of points $(x, u(x))$ with

$$
x_{n} \leq-\frac{\theta}{2}+H_{0}(u(x)), \quad\left|x^{\prime}\right| \leq \frac{l}{2}, \quad|u(x)| \leq \frac{1}{2}
$$

project along $e_{n}$ in a set of measure greater than $\left(1-(1-\bar{\mu})^{k_{0}}\right)\left|Q_{l / 2}\right|$. We show that if we choose $k_{0}$ large, universal such that

$$
c_{1} l^{n-1}>2(1-\bar{\mu})^{k_{0}}\left|Q_{l / 2}\right|
$$

and $\varepsilon$ small enough, then we obtain a contradiction.

As in Section 2 we use

$$
\begin{aligned}
\left(\frac{1}{2}|\nabla u|^{2}+h_{0}(u)\right) d x^{\prime} d x_{n} & \geq \sqrt{2 h_{0}(u)}\left|u_{n}\right| d x^{\prime} d x_{n} \\
& =\sqrt{2 h_{0}\left(x_{n+1}\right)} d x^{\prime} d x_{n+1} .
\end{aligned}
$$

Denote by

$$
A_{l}:=\left\{\left|x^{\prime}\right|<l\right\} \times\left\{\left|x_{n}\right|<l\right\} .
$$

Project along $e_{n}$ the points $(x, u(x))$, with $\left|x_{n}\right| \leq l$. From above we find that there exists a set of measure $c_{1} l^{n-1} / 2$ included in $Q_{l / 2}$ in which these points project twice. Using barrier functions it is not difficult to show that the projection covers the band

$$
\left\{\left|x_{n+1}\right|<1-c(l), \quad\left|x^{\prime}\right|<l\right\}, \quad \text { and } \quad c(l) \rightarrow 0 \text { as } l \rightarrow \infty .
$$

Hence we can find two small universal constants $c_{2}, c_{3}>0$ such that

$$
\begin{aligned}
J\left(u, A_{l}\right) & =\int_{A_{l}} \frac{1}{2}|\nabla u|^{2}+h_{0}(u) d x \\
& \geq \omega_{n-1} l^{n-1} \int_{c_{2}-1}^{1-c_{2}} \sqrt{2 h_{0}\left(x_{n+1}\right)} d x_{n+1}+\frac{c_{1}}{2}\left(\min _{|s| \leq 1 / 2} \sqrt{2 h_{0}(s)}\right) l^{n-1} \\
& \geq \omega_{n-1} l^{n-1} \int_{-1}^{1} \sqrt{2 h_{0}(s)} d s+c_{3} l^{n-1}
\end{aligned}
$$

where $\omega_{n-1}$ represents the volume of the $n-1$ dimensional unit sphere.

Assume by contradiction that there exist numbers $l_{k}, \theta_{k}$ with

$$
\theta_{k} l_{k}^{-1} \rightarrow 0, \quad \theta_{k} \geq \theta_{0},
$$

and local minimizers $u_{k}$ in $A_{2 l_{k}}$ satisfying the hypothesis of Theorem 7.3, and therefore also inequality (9.1). 
Denote by $\varepsilon_{k}:=l_{k}^{-1}$ and $v_{k}(x):=u_{k}\left(\varepsilon_{k}^{-1} x\right)$. From (9.1) we obtain

$$
\begin{aligned}
J_{\varepsilon_{k}}\left(v_{k}, A_{1}\right) & =\varepsilon_{k}^{n-1} J\left(u_{k}, A_{l_{k}}\right) \\
& \geq \omega_{n-1} \int_{-1}^{1} \sqrt{2 h_{0}(s)} d s+c_{3} .
\end{aligned}
$$

On the other hand, as $k \rightarrow \infty$ we have

$$
v_{k} \rightarrow \chi_{E}-\chi_{E^{c}} \text { in } L_{l o c}^{1}\left(A_{1}\right),
$$

where $E=A_{1} \cap\left\{x_{n}>0\right\}$. By Theorem 2.1 one has

$$
\lim _{k \rightarrow \infty} J_{\varepsilon_{k}}\left(v_{k}, A_{1}\right)=P_{A_{1}} E \int_{-1}^{1} \sqrt{2 h_{0}(s)} d s=\omega_{n-1} \int_{-1}^{1} \sqrt{2 h_{0}(s)} d s
$$

and we reach a contradiction.

With this Theorem 7.3 is proved.

\section{One-dimensional symmetry of global solutions}

In this section we use the results of Section 7 and obtain 1D symmetry for phase transitions from the 1D symmetry of global minimal surfaces. Recall Corollary 7.2.

Corollary. Let $u$ be a global minimizer of $J$ in $\mathbb{R}^{n}$. If the 0 level set $\{u=0\}$ is asymptotically flat at $\infty$, then $u$ is one-dimensional.

At the end of Section 4 we used the minimal surface theory to show that the level sets of minimizers of $J$ are asymptotically flat at $\infty$ in dimension $n \leq 7$. Using the corollary above we obtain

Theorem 10.1. A global minimizer of

$$
J(u)=\int \frac{1}{2}|\nabla u|^{2}+W(u) d x
$$

is one-dimensional if $n \leq 7$.

Next we prove the following version of De Giorgi's conjecture.

TheOrem 10.2. Let $u \in C^{2}\left(\mathbb{R}^{n}\right)$ be a solution of

$$
\triangle u=W^{\prime}(u)
$$

such that

$$
|u| \leq 1, \quad \partial_{n} u>0, \quad \lim _{x_{n} \rightarrow \pm \infty} u\left(x^{\prime}, x_{n}\right)= \pm 1 .
$$

Then $u$ is one-dimensional if $n \leq 8$.

Before we prove this Theorem we show that the \pm 1 limit assumption implies that $u$ is a global minimizer in $\mathbb{R}^{n}$.

Lemma 10.3. If $u$ satisfies (10.1), (10.2) then $u$ is a global minimizer for $J$. 


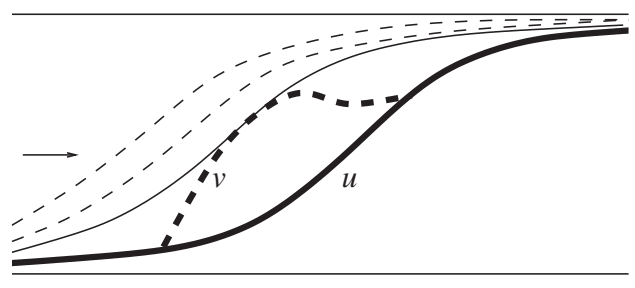

FIGURE 17. Sliding the graph of $u$

Proof. It suffices to show that in $B_{R}, u$ is the unique solution of

$$
\triangle v=W^{\prime}(v), \quad|v|<1, \quad v=u \quad \text { on } \partial B_{R} .
$$

Since

$$
\lim _{x_{n} \rightarrow \infty} u\left(x^{\prime}, x_{n}\right)=1 \text {, }
$$

we conclude that the graph of $u\left(x^{\prime}, x_{n}+t\right)$ is above the graph of $v$ for large $t$. We slide this graph in the $e_{n}$ direction till we touch $v$ for the first time (see Figure 17). From the Strong Maximum Principle we find that the first touching point occurs on $\partial B_{R}$. Since $u$ is strictly increasing in the $e_{n}$ direction, we can slide the graph of $u\left(x^{\prime}, x_{n}+t\right)$ till it coincides with the graph of $u$, hence $u \geq v$. Similarly we obtain $u \leq v$ which proves that $u$ is a global minimizer in $\mathbb{R}^{n}$.

Proof of Theorem 10.2. Assume $u(0)=0$ and define $u_{\varepsilon}(x)=$ $u\left(\varepsilon^{-1} x\right)$. Modica's theorem says that

$$
u_{\varepsilon_{k}} \rightarrow \chi_{E}-\chi_{\mathcal{C} E}
$$

holds for some sequence $\varepsilon_{k} \rightarrow 0$. Moreover, $u_{n}>0$ implies $E^{c}$ is a subgraph, hence $\partial E$ is a so-called quasi-solution in the $e_{n}$ direction (see chapters 16, 17 in the book of Giusti [40]). Then $\partial E$ is a hyperplane if $n \leq 8$ and the theorem follows from the corollary above.

If we assume that the 0 level set is a graph in the $x_{n}$ direction which has a one sided linear bound at $\infty$ then the conjecture is true in any dimension.

THEOREM 10.4. If u satisfies (10.1), (10.2) and

$$
\{u=0\} \subset\left\{x_{n}<C\left(1+\left|x^{\prime}\right|\right)\right\}
$$

then $u$ is one-dimensional.

Proof. As above, $u$ is a global minimizer. Then the limiting minimal surface has a linear bound by above and again we can conclude (see Giusti $[40])$ that that $\partial E$ is a hyperplane in any dimension. 


\section{De Giorgi's conjecture in $2 \mathrm{D}$ and $3 \mathrm{D}$}

In this section we present proofs to De Giorgi's conjecture in 2D and $3 \mathrm{D}$ which were first obtained by Ghoussoub and Gui [37] and Ambrosio and Cabre [5] respectively.

Our approach is due to Farina $[\mathbf{3 0 , 3 1}]$ and it is based on a calculation of Sternberg and Zumbrun $[\mathbf{6 2 , 6 3}]$. We follow it here since it closely resembles the one for minimal surfaces.

11.1. Minimal surfaces in 3D. Assume for simplicity that $\partial E$ is a smooth minimal surface in $\mathbb{R}^{n}$. Denote by $\nu_{x}$ the normal to the surface at $x$ and let $\phi$ be a function on $\partial E$ with compact support. If we perturb $\partial E$ by a map

$$
x \mapsto x+\varepsilon \phi(x) \nu_{x}
$$

then the area increases. By letting $\varepsilon \rightarrow 0$ we obtain (see Giusti [] for example) that the mean curvature is 0 at any point in $\partial E$ and moreover

$$
\int_{\partial E}\left(|\nabla \phi|^{2}-\phi^{2} \kappa^{2}\right) d \mathcal{H}^{n-1} \geq 0,
$$

where $\kappa^{2}$ represents the sum of the squares of the principal curvatures at $x \in \partial E$.

A smooth surface whose area increases under small local perturbations, i.e. has 0 mean curvature and satisfies the inequality above, is called a stable minimal surface.

One can obtain that a global minimal surface is a plane in $\mathbb{R}^{3}$ by choosing appropriate test functions $\phi$. Indeed, if we choose $\phi_{R}$ with compact support in $\bar{B}_{R}$ as

$$
\phi_{R}(x):=\left\{\begin{array}{l}
1, \quad|x| \leq 1 \\
\frac{1}{\log R} \log \frac{R}{|x|}, \quad 1<|x| \leq R
\end{array}\right.
$$

then

$$
\int_{\partial E}\left|\nabla \phi_{R}\right|^{2} d \mathcal{H}^{2} \leq(\log R)^{-2} \int_{\partial E \cap B_{R}}|x|^{-2} d \mathcal{H}^{2} .
$$

From the minimality of $\partial E$ we find that

$$
\mathcal{H}^{2}\left(\partial E \cap B_{r}\right) \leq C r^{2}
$$

hence

$$
\int_{\partial E \cap B_{R}}|x|^{-2} d \mathcal{H}^{2} \leq C \log R .
$$

In conclusion

$$
\int_{\partial E}\left|\nabla \phi_{R}\right|^{2} d \mathcal{H}^{2} \rightarrow 0 \quad R \rightarrow \infty
$$

which gives $\kappa=0$ in $\partial E \cap B_{1}$. This implies $\kappa \equiv 0$, i.e. $\partial E$ is a plane. 
We remark that Simons' proof that global minimal cones are planar up to dimension 7 , uses the stability condition by taking a test function $\phi(x)$ of the form $\kappa^{2} \psi(x)$.

11.2. Monotone solutions. First we check that monotone solutions of

$$
\triangle u=W^{\prime}(u)
$$

are stable, i.e. the energy increases under small compact perturbations. Precisely, we show that

$$
\int|\nabla \psi|^{2}+W^{\prime \prime}(u) \psi^{2} d x \geq 0, \quad \forall \psi \in C_{0}^{1}\left(\mathbb{R}^{n}\right) .
$$

Indeed, assume $u$ is increasing in the $e_{n}$ direction, i.e. $u_{n}>0$. Differentiating the equation we find

$$
\triangle u_{n}=W^{\prime \prime}(u) u_{n}
$$

We multiply by $\psi^{2} / u_{n}$ and integrate:

$$
\int \nabla \frac{\psi^{2}}{u_{n}} \nabla u_{n}+W^{\prime \prime}(u) \psi^{2} d x=0
$$

or

$$
\int|\nabla \psi|^{2}+W^{\prime \prime}(u) \psi^{2} d x=\int\left|\nabla \psi-\frac{\psi}{u_{n}} \nabla u_{n}\right|^{2} d x \geq 0
$$

As in the minimal surface case, we would like to perturb the level sets in the normal direction infinitesimally by a function $\phi(x)$. This corresponds to taking $\psi:=\phi|\nabla u|$ in the stability condition, i.e.

$$
\int|\nabla(\phi|\nabla u|)|^{2}+W^{\prime \prime}(u) \phi^{2}|\nabla u|^{2} d x \geq 0 .
$$

We obtain

$$
\int|\nabla \phi|^{2}|\nabla u|^{2}+\frac{1}{2} \nabla \phi^{2} \nabla\left(|\nabla u|^{2}\right)+\phi^{2}|\nabla(|\nabla u|)|^{2}+\phi^{2} W^{\prime \prime}(u)|\nabla u|^{2} d x \geq 0 .
$$

We integrate the second term by parts and use the equations for $u_{k}$,

$$
\begin{aligned}
\int \frac{1}{2} \nabla \phi^{2} \nabla\left(|\nabla u|^{2}\right) d x & =-\int \phi^{2} \triangle\left(\frac{1}{2}|\nabla u|^{2}\right) d x \\
& =-\int \phi^{2}\left(\left\|D^{2} u\right\|^{2}+W^{\prime \prime}(u)|\nabla u|^{2}\right) d x .
\end{aligned}
$$

By choosing a convenient system of coordinates it is not difficult to check that

$$
\left\|D^{2} u\right\|^{2}-\left.|\nabla| \nabla u\right|^{2}=\kappa^{2}|\nabla u|^{2}+\left|\nabla_{T}\right| \nabla u \|^{2}
$$


where $\nabla_{T}$ represents the tangential gradient along the level sets of $u$ and $\kappa^{2}(x)$ represents the sum of the squares of the principal curvatures on the level set of $u$ at $x$. In conclusion the stability inequality above simplifies as

$$
\int\left(|\nabla \phi|^{2}-\phi^{2} \kappa^{2}\right)|\nabla u|^{2} d x \geq 0
$$

This is precisely the stability condition for the minimal surface in which the $d \mathcal{H}^{n-1}$ measure on $\partial E$ is replaced by the weight $|\nabla u|^{2} d x$.

If the weight grows quadratically with the radius $R$ in $B_{R}$ i.e.

$$
\int_{B_{R}}|\nabla u|^{2} d x \leq C R^{2},
$$

then the same function $\phi_{R}$ gives as in the minimal surface case $\kappa \equiv 0$, that is $u$ is one-dimensional.

This inequality is always true in $\mathbb{R}^{2}$ since $L^{\infty}$ bounds for $\nabla u$ hold for bounded solutions under quite general assumptions on $W$, say for example $W$ is Lipschitz. Therefore in $\mathbb{R}^{2}$ we obtain one-dimensionality from stability which, as we saw above, is a weaker condition than monotonicity.

THEOREM 11.1 (Ghoussoub-Gui). Bounded stable global solution of

$$
\triangle u=W^{\prime}(u), \quad \text { in } \mathbb{R}^{2}
$$

are one-dimensional.

Ambrosio and Cabre proved that the quadratic bound holds also for monotone solutions in $\mathbb{R}^{3}$. As a consequence they obtain De Giorgi's conjecture in $\mathbb{R}^{3}$ :

Theorem 11.2 (Ambrosio-Cabre). Bounded monotone global solution of

$$
\triangle u=W^{\prime}(u), \quad \text { in } \mathbb{R}^{3}
$$

are one-dimensional.

We sketch the proof. From (4.1) we see that the quadratic bound for the energy holds for minimizers in $\mathbb{R}^{3}$. Hence, if the limits are \pm 1 as $x_{3} \rightarrow \pm \infty$ then we are done by Lemma 10.3. Otherwise, denote by $\underline{\mathrm{u}}\left(x^{\prime}\right), \bar{u}\left(x^{\prime}\right)$ the limits of $u\left(x^{\prime}, x_{3}\right)$ as $x_{3} \rightarrow \pm \infty$. It turns out that $\underline{\mathrm{u}}$ and $\bar{u}$ are stable solutions of the same equation in $\mathbb{R}^{2}$, and by the theorem above they are one-dimensional. From this the bound for the energy follows.

We finally mention that the quadratic growth cannot hold in dimension $n>3$ since it fails for the one-dimensional solution.

\section{Fully nonlinear equations}

The methods developed in Sections 7-10 are quite general and can be applied for other types of nonlinear, possibly degenerate elliptic equations. 
With Valdinoci and Sciunzi we proved in [66] the same theorems for the energy

$$
J_{p}(u, \Omega):=\int_{\Omega} \frac{1}{p}|\nabla u|^{p}+W(u) d x
$$

and the corresponding $p$-Laplace equation

$$
\triangle_{p} u=W^{\prime}(u), \quad \triangle_{p} u:=\operatorname{div}\left(|\nabla u|^{p-2}|\nabla u|\right) .
$$

In this section we present $1 D$ symmetry results for global solutions to fully nonlinear reaction-diffusion equations. We consider the equation $\mathbb{R}^{n}$

$$
F\left(D^{2} u\right)=f(u)
$$

where $F$ is uniformly elliptic with ellipticity constants $\lambda, \Lambda, F(0)=0$, and

$$
f \in C^{1}([-1,1]), \quad f( \pm 1)=0, \quad f^{\prime}(-1)>0, \quad f^{\prime}(1)>0 .
$$

The main assumption on $F$ and $f$ is that there exists a smooth increasing function $g_{0}: \mathbb{R} \rightarrow[-1,1]$ (one dimensional solution) such that

$$
\lim _{t \rightarrow \pm \infty} g_{0}(t)= \pm 1
$$

and $g_{0}$ solves the equation in all directions $\xi$, that is

$$
F\left(D^{2}\left(g_{0}(x \cdot \xi)\right)\right)=f\left(g_{0}(x \cdot \xi)\right)
$$

for every unit vector $\xi \in \mathbb{R}^{n}$. In other words, the compatibility condition says that the values of $F$ on the subset of rank one matrices $g_{0}^{\prime \prime}(t) \xi \otimes \xi$ equals $g\left(g_{0}(t)\right)$.

For example if $F$ is the maximal Pucci operator

$$
F(M)=\mathcal{M}_{\lambda, \Lambda}(M):=\max _{\lambda I \leq A \leq \Lambda} \operatorname{tr}(A \cdot M),
$$

and $f(s)=\max \left\{\Lambda\left(s^{3}-s\right), \lambda\left(s^{3}-s\right)\right\}$ with

$$
g_{0}(t)=\tanh \frac{t}{\sqrt{2}} .
$$

One difficulty of the problem is that, unlike the semilinear case, there is no variational formulation of the problem. Another difficulty consists in the fact that it is not clear whether the blow-downs of $\{u=s\}$ satisfy any equation. We will see that in general the level sets satisfy at large scales a curvature equation depending on $F, f$.

Together with De Silva we proved in [25] that De Giorgi's conjecture holds in two dimensions for these nonlinear equations.

TheOREM 12.1 (De Silva-Savin). Let $u \in C\left(\mathbb{R}^{2}\right)$ be a viscosity solution to (12.1) such that $|u| \leq 1$ and $u_{x_{2}}>0$. Then $u$ is one-dimensional. 
The condition $u_{x_{2}}>0$ can be relaxed to $m$ monotonicity in the $x_{2}$ direction i.e.

$$
u(x)<u\left(x+m e_{2}\right), \quad \text { for some } m>0 .
$$

The main step consists in proving that the sets $\{u<0\}$ and $\{u>0\}$ are convex. This is achieved by comparing $u$ with appropriate explicit barriers. Then one can deduce that the 0 level set is included in a strip of arbitrarily small width.

The higher dimensional problem was analyzed in [58]. As in the semilinear case, we considered monotone viscosity solutions of (12.1) which "connect" the constant solutions -1 and 1 at $\pm \infty$,

$$
u_{x_{n}}>0, \quad \lim _{x_{n} \rightarrow \pm \infty} u\left(x^{\prime}, x_{n}\right)= \pm 1
$$

Next result is a nonlinear version of the theorem of Modica. It says that if the blow-downs of the level sets converge to a limiting surface then this surface satisfies a curvature equation. This possible nonlinear curvature equation can be computed explicitly from $F$ and $f$.

TheOREM 12.2 (Limiting equation). Let u be a solution of (12.1), (12.2) and suppose that for a sequence $\varepsilon_{k} \rightarrow 0, \varepsilon_{k}\{u=0\}$ converges uniformly on compact sets to a surface $\Sigma$. Then, there exists a function $\tilde{G}$ depending on $F$ and $g_{0}$ such that

$$
\tilde{G}\left(\nu_{\Sigma}, I I_{\Sigma}\right)=0
$$

in the viscosity sense where $\nu_{\Sigma}(x), I I_{\Sigma}(x)$ represent the upward normal and the second fundamental form of $\Sigma$ at a point $x \in \Sigma$. The function $\tilde{G}(\xi, \cdot)$ is defined on the space of $n \times n$ symmetric matrices $M$ with $M \xi=0$, is homogenous of degree one, and uniformly elliptic.

The function $\tilde{G}$ is linear in the second argument and it depends on the derivatives of $F$ on the $n$ dimensional cone $\xi \otimes \xi, \xi \in \mathbb{R}^{n}$.

If $F$ is invariant under rotations and $F \in C^{1}$ then $\tilde{G}(\xi, M)=\operatorname{tr} M$, i.e. $\Sigma$ is a minimal graph as in the semilinear case. If for example $F=\mathcal{M}_{\lambda, \Lambda}^{+}$ then $\tilde{G}$ is nonlinear, $\tilde{G}(\xi, \cdot)=\mathcal{M}_{\lambda, \Lambda}^{-}$.

The methods developed in Sections 7-9 generalize to this setting. Due to the monotonicity assumption, the Harnack inequality requires only a onesided bound.

THEOREM 12.3 (Harnack inequality). Let $u$ be a solution of (12.1), (12.2) with

$$
\{u=0\} \cap\left\{\left|x^{\prime}\right|<l\right\} \subset\left\{x \cdot \xi_{0}>0\right\},
$$

for some unit vector

$$
\left|\xi_{0}\right|=1, \quad \angle\left(\xi_{0}, e_{n}\right) \leq \beta<\pi / 2 .
$$

Assume

$$
(0, \theta) \in\{u=0\}, \quad \theta \geq \theta_{0}
$$


for some fixed $\theta_{0}$. If $\theta / l \leq \varepsilon\left(\theta_{0}\right)$ then

$$
\{u=0\} \cap\left\{\left|x^{\prime}\right|<l / 2\right\} \subset\left\{x \cdot \xi_{0}<K \theta\right\},
$$

where the constant $K$ depends only on $\lambda, \Lambda, n, f, g_{0}, \beta$ and the constant $\varepsilon\left(\theta_{0}\right)>0$ depends on the previous constants and $\theta_{0}$.

As a consequence of Harnack inequality we obtain a Liouville theorem for the level sets.

THEOREM 12.4. If $\{u=0\}$ is above (in the $e_{n}$ direction) a plane $\{x \cdot \xi=$ $0\}$, then $u$ is one-dimensional.

A consequence of Theorem 12.4 is a proof of the Gibbons conjecture for equation (12.1). We recall that the conjecture states that global solutions are one-dimensional if the limits in (12.2) are uniform in $x^{\prime}$.

If we assume more regularity on the operator $F$ then we can use Theorem 12.3 and show the improvement of flatness for the level sets.

THEOREM 12.5 (Improvement of flatness). Let $u$ be a solution of (12.1), (12.2). Assume that $F \in C^{1}$ and

$$
\begin{gathered}
0 \in\{u=0\} \cap\left\{\left|x^{\prime}\right|<l\right\} \subset\left\{\left|x \cdot \xi_{0}\right|<\theta\right\}, \\
\left|\xi_{0}\right|=1, \quad \angle\left(\xi_{0}, e_{n}\right) \leq \beta<\pi / 2, \quad \theta \geq \theta_{0} .
\end{gathered}
$$

If $\theta / l \leq \varepsilon\left(\theta_{0}\right)$ then, for some unit vector $\xi_{1}$

$$
\{u=0\} \cap\left\{\left|x^{\prime}\right|<\eta_{2} l\right\} \subset\left\{\left|x \cdot \xi_{1}\right|<\eta_{1} \theta\right\},
$$

where the constants $0<\eta_{1}<\eta_{2}<1$ depend only on $\lambda, \Lambda$, $n$ and the constant $\varepsilon\left(\theta_{0}\right)$ depends on $F, f, \beta$ and $\theta_{0}$.

As a consequence of the theorem above we obtain $1 D$ symmetry of global solutions which have one Lipschitz level set.

TheOrem 12.6. Assume that $F \in C^{2}$, u solves (12.1), (12.2) and $\{u=$ $0\}$ is a Lipschitz graph in the $e_{n}$ direction. Then $u$ is one dimensional.

\section{References}

[1] Alberti G., Ambrosio L., Cabre X., On a long-standing conjecture of E. De Giorgi: symmetry in 3D for general nonlinearities and a local minimality property. Acta Appl. Math. 65 (2001), no. 1-3, 9-33.

[2] Almgren F.J., The theory of varifolds- a variational calculus in the large for the $k$-dimensinal area integrand. Mimeographed notes, Princeton, 1965.

[3] Almgren F.J., Existence and regularity almost everywhere of solutions to elliptic variational problems among surfaces of varying topological type and singularity structure. Ann. of Math. (2) 87 (1968), 321-391.

[4] Alt H.W., Caffarelli L., Existence and regularity for a minimum problem with free boundary. J. Reine Angew. Math 325 (1981),105-144.

[5] Ambrosio L., Cabre X., Entire solutions of semilinear elliptic equations in $\mathbb{R}^{3}$ and a conjecture of De Giorgi. J. American Math. Soc. 13, (2000), 725-739. 
[6] Baldo S., Minimal interface criterion for phase transitions in mixtures of Cahn-Hilliard fluids. Ann. Inst. H. Poincar Anal. Non Linaire7 (1990), no. 2, 67-90.

[7] Barlow M., Bass R., Gui C., The Liouville property and a conjecture of De Giorgi. Comm. Pure Appl. Math. 53 (2000), no. 8, 1007-1038.

[8] Berestycki H., Caffarelli L., Nirenberg L., Further qualitative properties for elliptic equations in unbounded domains. Ann. Scuola Norm. Sup. Pisa Cl. Sci. (4) 25 (1997), no. 1-2, 69-94 (1998).

[9] Berestycki H., Hamel F., Monneau, R., One-dimensional symmetry of bounded entire solutions of some elliptic equations. Duke Math. J. 103 (2000), no. 3, 375-396.

[10] Bernstein S., Sur un theoreme de geometrie et son application aux equationes aux derivees partielles du type elliptique. Comm. Soc. Math. de Kharkov (2) 15 (19151917), 38-45. German transl.:Math. Z. 26 (1927), 551-558.

[11] Bombieri E., De Giorgi E., Giusti E., Minimal cones and the Bernstein problem. Invent. Math. 7 (1969), 243-268.

[12] Cahn J., Hilliard J., Free energy of a nonuniform system I. Interfacial free energy. J. Chem. Phys 28 (1958), 258-267.

[13] Cabre X., Terra J., Saddle-shaped solutions of bistable diffusion equations in all of $\mathbb{R}^{2 m}$. Preprint, arXiv:0801.3379.

[14] Cabre X., Terra J., Qualitative properties of saddle-shaped solutions to bistable diffusion equations. Preprint, arXiv:0907.3008.

[15] Caffarelli L., Cabre X., Fully Nonlinear Elliptic Equations. Colloquium Publications 43, American Mathematical Society, Providence, RI, (1995).

[16] Caffarelli L., Cordoba A., An elementary regularity theory of minimal surfaces. Differential Integral Equations 6 (1993), no. 1, 1-13.

[17] Caffarelli L., Cordoba A., Uniform convergence of a singular perturbation problem. Comm. Pure Appl. Math. 48 (1995), no. 1, 1-12.

[18] Caffarelli L., Cordoba A., Phase transitions: uniform regulariy of the intermediate layers. J. Reine Angew. Math. 593 (2006), 209-235.

[19] Caffarelli L., Garofalo N., Segàla F., A gradient bound for entire solutions of quasilinear equations and its consequences. Comm. Pure Appl. Math. 47 (1994), no. 11, $1457-1473$.

[20] De Giorgi E., Convergence problems for functional and operators. Proc. Int. Meeting on Recent Methods in Nonlinear Analysis (Rome, 1978), 131-188.

[21] De Giorgi E., Su una teoria generale della misura $(r-1)$-dimensionale in uno spazio ad $r$ dimensioni. Ann. Mat.Pura Appl. (4) 36 (1954), 191-213.

[22] De Giorgi E., Nuovi teoremi relativi alle misure $(r-1)$-dimensionale inn uno spazio ad $r$ dimensioni. Ricerche Mat. 4 (1955), 95-113.

[23] De Giorgi E., Complimenti alla teoria della misura $(n-1)$-dimensionale in uno spazio n-dimensionale. Sem. Mat. Scuola Norm. Sup. Pisa, 1960-61 . Editrice Tecnico Scientifica, Pisa, (1961).

[24] De Giorgi E., Frontiere orientate di misura minima. Sem. Mat. Scuola Norm. Sup. Pisa 1960 Editrice Tecnico Scientifica, Pisa 1961.

[25] De Silva D., Savin O., Symmetry of global solutions to a class of fully nonlinear elliptic equations in 2D. Indiana Univ. Math. J., 58 (1), (2009), 301-315.

[26] Del Pino M., Kowalczyk M., Wei J., On De Giorgi Conjecture in dimension $N \geq 9$. Preprint, arXiv:0806.3141.

[27] Douglas J., Solution of the problem of Plateau. Trans. Amer. Math. Soc. 33 (1931), 263-321.

[28] Farina A., Some remarks on a conjecture of De Giorgi. Calc. Var. Partial Differential Equations 8 (1999), 129-154.

[29] Farina A., Symmetry for solutions of semilinear elliptic equations in $\mathbb{R}^{n}$ and related conjectures. Ricerche Mat. 48 (1999), 129-154. 
[30] Farina A., Propietes qualitatives de solutions d'equations et systemes d'equations non-lineaires. Habilitation a diriger des recherches 2002 Paris VI.

[31] Farina A., Sciunzi B., Valdinoci E., Bernstein and De Giorig type problems: new results via a geometric approach. Preprint (2007).

[32] Federer H., Some properties of distributions whose partial derivatives are represented by integration. Bull. Amer. Math. Soc. 74 (1968), 183-186.

[33] Federer H., Geometric measure theory. Springer-Verlag, Berlin-Heidelberg-New York, (1969).

[34] Federer H., The singular set of area minimizing rectifiable currents with codimension one and of area minimizing flat chains modulo two with arbitrary codimension. Bull. Amer. Math. Soc. 76 (1970), 767-771.

[35] Fleming W.H., On the oriented Plateau problem. Rend. Circ. Mat. Palermo (2) (1962) 69-90.

[36] Fonseca I., Tartar L., The gradient theory of phase transitions for systems with two potential wells. Proc. Roy. Soc. Edinburgh Sect. A 111 (1989), no. 1-2, 89-102.

[37] Ghoussoub N., Gui C., On a conjecture of De Giorgi and some related problems. Math. Ann. 311 (1998), 481-491.

[38] Ghoussoub N., Gui C., On De Giorgi's conjecture in dimensions 4 and 5. Ann. of Math. (2) 157 (2003), no. 1, 313-334.

[39] Gilbarg D., Trudinger N., Elliptic Partial Differential Equations of second order. Springer-Verlag, New York, (1983).

[40] Giusti E., Minimal Surfaces and functions of bounded variation. Birkhauser Verlag, Basel-Boston (1984).

[41] Jerison D., Monneau R., The existence of a symmetric global minimizer on $\mathbb{R}^{n-1}$ implies the existence of a counter-example to a conjecture of De Giorgi in $\mathbb{R}^{n}$. C. R. Acad. Sci. Paris Sr. I Math . 333 (2001), no. 5, 427-431.

[42] Kawohl B., Symmetry or not? Math. Intelligencer 20 (1998), 16-22.

[43] Kohn R., Sternberg P., Local minimisers and singular perturbations. Proc. Roy. Soc. Edinburgh Sect. A 111 (1989), no. 1-2, 69-84.

[44] Krylov N., Safonov M., A certain property of solutions of parabolic equations with measurable coefficients. Izv. Akad. Nauk SSSR Ser. mat., 44 (1980), 161-175.

[45] Modica L., $\Gamma$-convergence to minimal surfaces problem and global solutions of $\Delta u=$ $2\left(u^{3}-u\right)$. Proceedings of the International Meeting on Recent Methods in Nonlinear Analysis (Rome, 1978), pp. 223-244, Pitagora, Bologna, (1979).

[46] Modica L., A gradient bound and a Liouville theorem for nonlinear Poisson equations. Comm. Pure Appl. Math. 38 (1985), no. 5, 679-684.

[47] Modica L., The gradient theory of phase transitions and the minimal interface criterion. Arch. Rational Mech. Anal. 98 (1987), no. 2, 123-142.

[48] Modica L., Monotonicity of the energy for entire solutions of semilinear elliptic equations. Partial differential equations and the calculus of variations, Vol. II, 843-850, Progr. Nonlinear Differential Equations Appl., 2, Birkhuser Boston, Boston, MA, (1989).

[49] Modica L., Gradient theory of phase transitions with boundary contact energy. Ann. Inst. H. Poincar Anal. No Lineaire 4 (1987), no. 5, 487-512.

[50] Modica L., Mortola S., Some entire solutions in the plane of nonlinear Poisson equations. Boll. Un. Mat. Ital. B (5) 17 (1980), no. 2, 614-622.

[51] Modica L., Mortola S., Un esempio di $\Gamma$-convergenza. (Italian) Boll. Un. Mat. Ital. B (5) 14 (1977), no. 1, 285-299.

[52] Owen N., Nonconvex variational problems with general singular perturbations. Trans. Amer. Math. Soc. 310 (1988), no. 1, 393-404.

[53] Rado T., The problem of the least area and the problem of Plateau. Math. Z. 32 (1930), 763-796.

[54] Rado T., On the problem of Plateau, Springer-verlag, Berlin, (1933). 
[55] Savin O., Phase transitions: Regularity of flat level sets. PhD Thesis UT Austin, (2003).

[56] Savin O., Regularity of flat level sets in phase transitions. Ann. of Math. (2) 169 (2009), no.1, 41-78.

[57] Savin O., Small perturbation solutions for elliptic equations. Comm. Partial Differential Equations 32 (2007), 557-578.

[58] Savin O., Entire solutions to a class of fully nonlinear elliptic equations. Ann. Sc. Norm. Super. Pisa Cl. Sci. (5), 7 (2008), no.3, 369-405.

[59] Simons J., Minimal varieties in riemannian manifolds. Ann. of Math. (2) 88 (1968), $62-105$.

[60] Sternberg P., The effect of a singular perturbation on nonconvex variational problems. Arch. Rational Mech. Anal. 101 (1988), no. 3, 209-260.

[61] Sternberg P., Vector-valued local minimizers of nonconvex variational problems. Rocky Mountain J. Math. 21 (1991), no. 2, 799-807.

[62] Sternberg P., Zumbrun K., Connectivity of phase boundaries in a strictly convex domain. Arch. rational Mech. Anal. 141 (1998), 375-400.

[63] Sternberg P., Zumbrun K., A Poincare inequality with applications to volume constrained area minimizing surfaces. J. Reine Angew. Math. 503 (1998), 63-85.

[64] Tonegawa Y., Phase field model with a variable chemical potential. Proc. Roy. Soc. Edinburgh Sect. A 132 (2002), no. 4, 993-1019.

[65] Tonegawa Y., Domain dependent monotonicity formula for a singular perturbation problem. Indiana Univ. Math. J. 52 (2003), no. 1, 69-83.

[66] Valdinoci E., Sciunzi B., Savin O, Flat level set regularity of $p$-Laplace phase transitions. Mem. Amer. Math. Soc. 182 (2006), no. 858, vi+144pp.

Department of Mathematics, Columbia University, New York, NY $10027^{1}$

E-mail address: savin@math.columbia.edu 
